Computer Applications: An International Journal (CAIJ), Vol.2, No.1, February 2015

\title{
ON ANALYTICAL APPROACH FOR ANALYSIS INFLUENCE OF MISMATCH-INDUCED STRESS IN A HETEROSTRUC- TURE ON DISTRIBUTIONS OF CONCENTRATIONS OF DO- PANTS IN A MULTIEMITTER HETEROTRANSISTOR
}

\author{
E.L. Pankratov ${ }^{1,3}$, E.A. Bulaeva ${ }^{1,2}$ \\ ${ }^{1}$ Nizhny Novgorod State University, Russia \\ ${ }^{2}$ Nizhny Novgorod State University of Architecture and Civil Engineering, Russia \\ ${ }^{3}$ Nizhny Novgorod Academy of the Ministry of Internal Affairs of Russia, Russia
}

\begin{abstract}
In this paper we analytically model technological processes of manufacturing of multiemitter heterotransistors with account relaxation of mismatch-induced stress. Based on this modeling some recommendations to increase sharpness of p-n-junctions, which included into the transistors, and increasing compactness of the transistors have been formulated.
\end{abstract}

\section{KEYWORDS}

Multiemitter heterotransistors, distributions of concentrations, influence of mismatch-induced stress, analytical approach to model technological processes

\section{INTRODUCTION}

In the present time integration degree of elements of integrated circuits is intensively increasing ( $p$ - $n$-junctions, field-effect and bipolar transistors, ...) [1-14]. At the same time with increasing of integration degree of elements of integrated circuits dimensions of this elements decreases. It is attracted an interest increasing of performance of the above elements [1-7]. To increase the performance it should be determine materials with higher values of charge carrier mobility for manufacturing elements of integrated circuits [15-18]. Another way to increase the performance is development of new and optimization of existing technological processes. It could be used different approaches to decrease dimensions of elements of integrated circuits. Two of them are laser and microwave types of annealing of dopants and/or radiation defects [19-21]. Using this approaches leads to manufacture inhomogenous distribution of temperature in the considered materials. Inhomogeneity of the distribution gives us possibility to decrease dimensions of elements of integrated circuits and their discrete analogs. Another way to decrease dimensions of the elements is doping of required parts of epitaxial layers of heterostructures by diffusion or ion implantation. However using the approach leads to necessity to optimize annealing of dopant and /or radiation defects $[22,23]$. It is also attracted an interest radiation processing of doped materials. The processing leads to changing of distributions of concentrations of dopants in doped materials [24]. The changing could leads to decreasing dimensions of the above elements [25].

In this paper we consider manufacturing of multiemitter transistor in the heteristructure, which consist of a substrate and three epitaxial layers (see Fig. 1). Some sections have been manufactured in the epitaxial layers by using another materials (see Fig. 1). After manufacturing these section they are should be doped by diffusion or ion implantation. The doping is necessary to produce required type of conductivity in the sections $(n$ or $p)$. The first step of manufacturing of the transistor is formation the first epitaxial layer (nearest to the substrate). After finishing of the formation is doping of the single section by diffusion or ion implantation. Farther we consider DOI : $10.5121 /$ caij.2015.2107 
manufacturing the second epitaxial layer with own section. After that we consider doping of this section by diffusion or ion implantation. Farther we consider manufacturing the third epitaxial layer with several sections. After that we consider doping of these sections by diffusion or ion implantation. Farther the dopants and/or radiation defects should be annealed. Analysis of dynamics of redistribution of dopants and radiation defects in the considered heterostructure with account relaxation of mechanical stress is the main aim of the present paper.

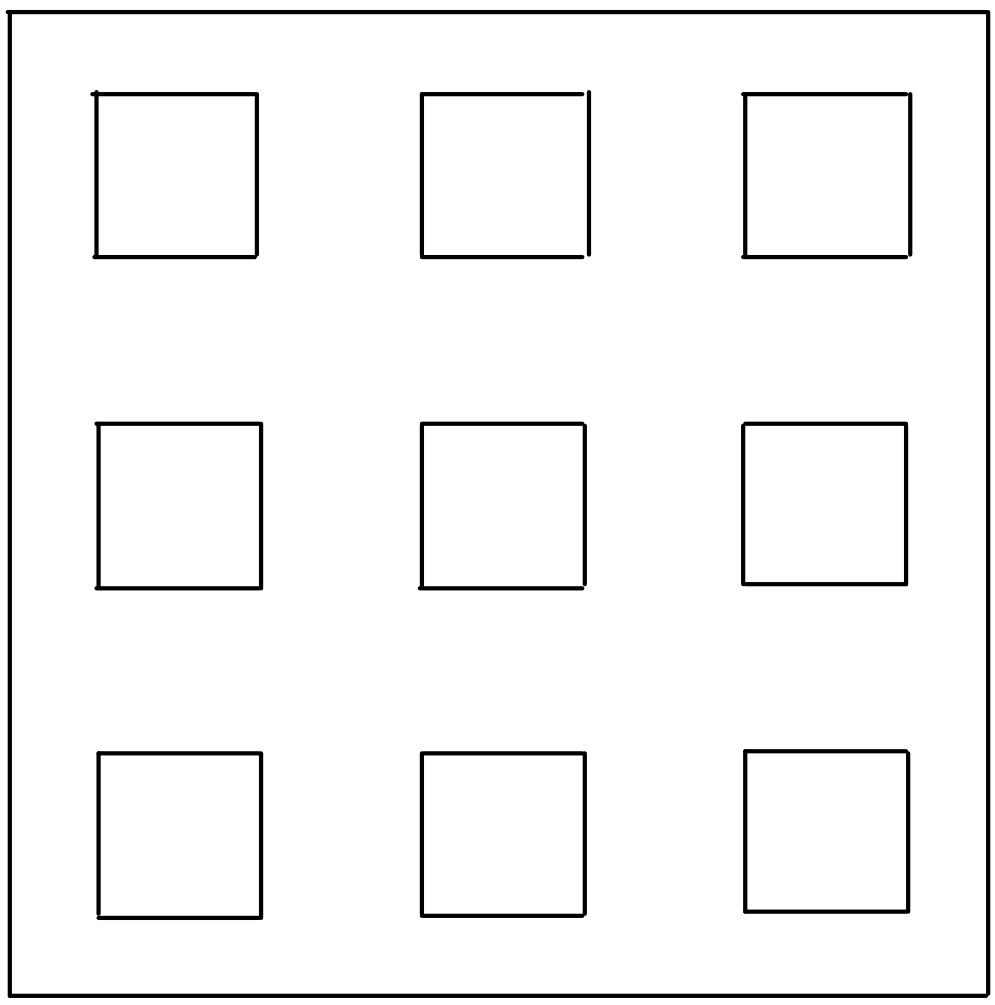

Fig. 1a. Structure of the considered heterostructure. The heterostructure include into itself a substrate and three epitaxial layers with sections, manufactured by using another materials. View from top

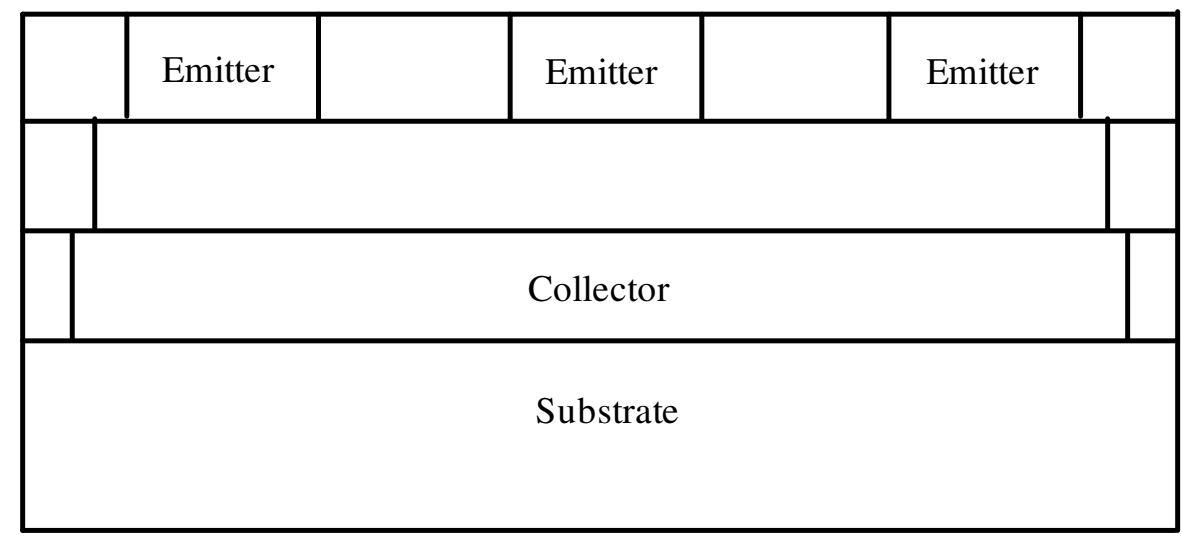

Fig. 1b. Heterostructure, which consist of a substrate and three epitaxial layers with sections, manufactured by using another materials. View from one side 


\section{Method of solution}

To solve our aim we shall analyze spatio-temporal distribution of concentration of dopant. We determine the spatio-temporal distribution of concentration of dopant by solving the following boundary problems $[1,3-5,26,27]$

$$
\begin{aligned}
\frac{\partial C(x, y, z, t)}{\partial t}= & \frac{\partial}{\partial x}\left[D \frac{\partial C(x, y, z, t)}{\partial x}\right]+\frac{\partial}{\partial y}\left[D \frac{\partial C(x, y, z, t)}{\partial y}\right]+\frac{\partial}{\partial z}\left[D \frac{\partial C(x, y, z, t)}{\partial z}\right]+ \\
& +\Omega \frac{\partial}{\partial x}\left[\frac{D_{S}}{k T} \nabla_{S} \mu(x, y, z, t) \int_{0}^{L_{z}} C(x, y, W, t) d W\right]+ \\
& +\Omega \frac{\partial}{\partial y}\left[\frac{D_{S}}{k T} \nabla_{S} \mu(x, y, z, t) \int_{0}^{L} C(x, y, W, t) d W\right]
\end{aligned}
$$

with initial and boundary conditions

$$
\begin{gathered}
C(x, y, z, 0)=f_{C}(x, y, z),\left.\frac{\partial C(x, y, z, t)}{\partial x}\right|_{x=0}=0,\left.\frac{\partial C(x, y, z, t)}{\partial x}\right|_{x=L_{x}}=0,\left.\frac{\partial C(x, y, z, t)}{\partial y}\right|_{y=0}=0, \\
\left.\frac{\partial C(x, y, z, t)}{\partial y}\right|_{x=L_{y}}=0,\left.\frac{\partial C(x, y, z, t)}{\partial z}\right|_{z=0}=0,\left.\frac{\partial C(x, y, z, t)}{\partial z}\right|_{x=L_{z}}=0 .
\end{gathered}
$$

The function $C(x, y, z, t)$ describes the spatio-temporal distribution of concentration of dopant; $\Omega$ is the atomic volume of the dopant; symbol $\nabla_{S}$ is the surficial gradient; the function $\int_{0}^{L_{z}} C(x, y, z, t) d z$ describes the surficial concentration of dopant in area of interface between materials of heterostructure. In this case we assume, that Z-axis is perpendicular to interface between materials of heterostructure; $\mu(x, y, z, t)$ is the chemical potential; $D$ are $D_{S}$ are the coefficients of volumetric and surficial diffusions (the surficial diffusion is the consequences of mismatch induced stress). Values of diffusion coefficients depend on properties of materials of heterostructure, speed of heating and cooling of heterostructure, concentration of dopant. Dependences of dopant diffusion coefficients on parameters could be approximated by the following relations [3,28-30]

$$
\begin{gathered}
D_{C}=D_{L}(x, y, z, T)\left[1+\xi \frac{C^{\gamma}(x, y, z, t)}{P^{\gamma}(x, y, z, T)}\right]\left[1+\varsigma_{1} \frac{V(x, y, z, t)}{V^{*}}+\varsigma_{2} \frac{V^{2}(x, y, z, t)}{\left(V^{*}\right)^{2}}\right], \\
D_{S}=D_{S L}(x, y, z, T)\left[1+\xi_{S} \frac{C^{\gamma}(x, y, z, t)}{P^{\gamma}(x, y, z, T)}\right]\left[1+\varsigma_{1} \frac{V(x, y, z, t)}{V^{*}}+\varsigma_{2} \frac{V^{2}(x, y, z, t)}{\left(V^{*}\right)^{2}}\right] .
\end{gathered}
$$

Functions $D_{L}(x, y, z, T)$ and $D_{L S}(x, y, z, T)$ describe the spatial (due to varying of values of dopant diffusion coefficients in the heterostruicture with varying of coordinate) and temperature (due to Arrhenius law) dependences of dopant diffusion coefficients; $T$ is the temperature of annealing; the function $P(x, y, z, T)$ describe the limit of solubility of dopant; parameter $\gamma$ could be integer in the following interval $\gamma \in[1,3]$ [3] and varying with variation of properties of materials of heterostructure; function $V(x, y, z, t)$ describes the spatio-temporal distribution of concentration of radia- 
tion vacancies; $V^{*}$ is the equilibrium distribution of vacancies. Detailed description of concentrational dependence of dopant diffusion coefficient is presented in [3]. We determine the spatiotemporal distributions of concentration of point radiation defects by solving the following system of equations [28-30]

$$
\begin{gathered}
\frac{\partial I(x, y, z, t)}{\partial t}=\frac{\partial}{\partial x}\left[D_{I}(x, y, z, T) \frac{\partial I(x, y, z, t)}{\partial x}\right]+\frac{\partial}{\partial y}\left[D_{I}(x, y, z, T) \frac{\partial I(x, y, z, t)}{\partial y}\right]+ \\
+\frac{\partial}{\partial z}\left[D_{I}(x, y, z, T) \frac{\partial I(x, y, z, t)}{\partial z}\right]-k_{I, I}(x, y, z, T) I^{2}(x, y, z, t)-k_{I, V}(x, y, z, T) I(x, y, z, t) \times \\
\times V(x, y, z, t)+\Omega \frac{\partial}{\partial x}\left[\frac{D_{I S}}{k T} \nabla_{S} \mu(x, y, z, t) \int_{0}^{L_{z}} I(x, y, W, t) d W\right]+ \\
+\Omega \frac{\partial}{\partial y}\left[\frac{D_{I S}}{k T} \nabla_{S} \mu(x, y, z, t) \int_{0}^{L_{z}} I(x, y, W, t) d W\right](3) \\
\frac{\partial V(x, y, z, t)}{\partial t}=\frac{\partial}{\partial x}\left[D_{V}(x, y, z, T) \frac{\partial V(x, y, z, t)}{\partial x}\right]+\frac{\partial}{\partial y}\left[D_{V}(x, y, z, T) \frac{\partial V(x, y, z, t)}{\partial y}\right]+ \\
+\frac{\partial}{\partial z}\left[D_{V}(x, y, z, T) \frac{\partial V(x, y, z, t)}{\partial z}\right]-k_{V, V}(x, y, z, T) V^{2}(x, y, z, t)-k_{I, V}(x, y, z, T) I(x, y, z, t) \times \\
\times V(x, y, z, t)+\Omega \frac{\partial}{\partial x}\left[\frac{D_{V S}}{k T} \nabla_{S} \mu(x, y, z, t) \int_{0}^{L_{z}} V(x, y, W, t) d W\right]+ \\
+\Omega \frac{\partial}{\partial y}\left[\frac{D_{V S}}{k T} \nabla_{S} \mu(x, y, z, t) \int_{0}^{L_{z}} V(x, y, W, t) d W\right]
\end{gathered}
$$

with boundary and initial conditions

$$
\begin{gathered}
\left.\frac{\partial I(x, y, z, t)}{\partial x}\right|_{x=0}=0,\left.\frac{\partial I(x, y, z, t)}{\partial x}\right|_{x=L_{x}}=0,\left.\frac{\partial I(x, y, z, t)}{\partial y}\right|_{y=0}=0,\left.\frac{\partial I(x, y, z, t)}{\partial y}\right|_{y=L_{y}}=0, \\
\left.\frac{\partial I(x, y, z, t)}{\partial z}\right|_{z=0}=0,\left.\frac{\partial I(x, y, z, t)}{\partial z}\right|_{z=L_{z}}=0,\left.\frac{\partial V(x, y, z, t)}{\partial x}\right|_{x=0}=0,\left.\frac{\partial V(x, y, z, t)}{\partial x}\right|_{x=L_{x}}=0, \\
\left.\frac{\partial V(x, y, z, t)}{\partial y}\right|_{y=0}=0,\left.\frac{\partial V(x, y, z, t)}{\partial y}\right|_{y=L_{y}}=0,\left.\frac{\partial V(x, y, z, t)}{\partial z}\right|_{z=0}=0,\left.\frac{\partial V(x, y, z, t)}{\partial z}\right|_{z=L_{z}}=0, \\
I(x, y, z, 0)=f_{I}(x, y, z), V(x, y, z, 0)=f_{V}(x, y, z) .
\end{gathered}
$$

Function $I(x, y, z, t)$ describes the spatio-temporal distribution of concentration of radiation interstitials; $I^{*}$ is the equilibrium distribution of interstitials; $D_{I}(x, y, z, T), D_{V}(x, y, z, T), D_{I S}(x, y, z, T)$, $D_{V S}(x, y, z, T)$ are the coefficients of volumetric and surficial diffusions of interstitials and vacancies, respectively; terms $V^{2}(x, y, z, t)$ and $I^{2}(x, y, z, t)$ correspond to generation of divacancies and diinterstitials, respectively (see, for example, [30] and appropriate references in this book); $k_{I, V}(x, y, z, T)$ and $k_{I, I}(x, y, z, T)$ are the parameters of recombination of point radiation defects, $k_{V, V}(x$, $y, z, T)$ is the parameter generation of their complexes.

We determine spatio-temporal distributions of concentrations of divacancies $\Phi_{V}(x, y, z, t)$ and diinterstitials $\Phi_{I}(x, y, z, t)$ by solving the following boundary problem [28-30] 
Computer Applications: An International Journal (CAIJ), Vol.2, No.1, February 2015

$$
\begin{gathered}
\frac{\partial \Phi_{I}(x, y, z, t)}{\partial t}=\frac{\partial}{\partial x}\left[D_{\Phi_{I}}(x, y, z, T) \frac{\partial \Phi_{I}(x, y, z, t)}{\partial x}\right]+\frac{\partial}{\partial y}\left[D_{\Phi_{I}}(x, y, z, T) \frac{\partial \Phi_{I}(x, y, z, t)}{\partial y}\right]+ \\
+\frac{\partial}{\partial z}\left[D_{\Phi_{I}}(x, y, z, T) \frac{\partial \Phi_{I}(x, y, z, t)}{\partial z}\right]+\Omega \frac{\partial}{\partial x}\left[\frac{D_{\Phi_{I} S}}{k T} \nabla_{S} \mu(x, y, z, t) \int_{0}^{L_{z}} \Phi_{I}(x, y, W, t) d W\right]+ \\
+\Omega \frac{\partial}{\partial y}\left[\frac{D_{\Phi I S}}{k T} \nabla_{S} \mu(x, y, z, t) \int_{0}^{L_{z}} \Phi_{I}(x, y, W, t) d W\right]+k_{I, I}(x, y, z, T) I^{2}(x, y, z, t)+ \\
+k_{I}(x, y, z, T) I(x, y, z, t) \\
\begin{array}{c}
\frac{\partial \Phi_{V}(x, y, z, t)}{\partial t}=\frac{\partial}{\partial x}\left[D_{\Phi_{V}}(x, y, z, T) \frac{\partial \Phi_{V}(x, y, z, t)}{\partial x}\right]+\frac{\partial}{\partial y}\left[D_{\Phi_{V}}(x, y, z, T) \frac{\partial \Phi_{V}(x, y, z, t)}{\partial y}\right]+ \\
+\frac{\partial}{\partial z}\left[D_{\Phi_{V}}(x, y, z, T) \frac{\partial \Phi_{V}(x, y, z, t)}{\partial z}\right]+\Omega \frac{\partial}{\partial x}\left[\frac{D_{\Phi_{V} S}}{k T} \nabla_{S} \mu(x, y, z, t) \int_{0}^{L_{z}} \Phi_{V}(x, y, W, t) d W\right]+ \\
+\Omega \frac{\partial}{\partial y}\left[\frac{D_{\Phi_{V} S}}{k T} \nabla_{S} \mu(x, y, z, t) \int_{0}^{L_{z}} \Phi_{V}(x, y, W, t) d W\right]+k_{V, V}(x, y, z, T) V^{2}(x, y, z, t)+ \\
+k_{V}(x, y, z, T) V(x, y, z, t)
\end{array}
\end{gathered}
$$

with boundary and initial conditions

$$
\begin{gathered}
\left.\frac{\partial \Phi_{I}(x, y, z, t)}{\partial x}\right|_{x=0}=0,\left.\frac{\partial I(x, y, z, t)}{\partial x}\right|_{x=L_{x}}=0,\left.\frac{\partial I(x, y, z, t)}{\partial y}\right|_{y=0}=0,\left.\frac{\partial I(x, y, z, t)}{\partial y}\right|_{y=L_{y}}=0, \\
\left.\frac{\partial \Phi_{I}(x, y, z, t)}{\partial z}\right|_{z=0}=0,\left.\frac{\partial I(x, y, z, t)}{\partial z}\right|_{z=L_{z}}=0,\left.\frac{\partial \Phi_{V}(x, y, z, t)}{\partial x}\right|_{x=0}=0,\left.\frac{\partial V(x, y, z, t)}{\partial x}\right|_{x=L_{x}}=0, \\
\left.\frac{\partial V(x, y, z, t)}{\partial y}\right|_{y=0}=0,\left.\frac{\partial V(x, y, z, t)}{\partial y}\right|_{y=L_{y}}=0,\left.\frac{\partial V(x, y, z, t)}{\partial z}\right|_{z=0}=0,\left.\frac{\partial \Phi_{V}(x, y, z, t)}{\partial z}\right|_{z=L_{z}}=0, \\
\Phi_{I}(x, y, z, 0)=f_{\Phi I}(x, y, z), \Phi_{V}(x, y, z, 0)=f_{\Phi V}(x, y, z) .
\end{gathered}
$$

Here $D_{\Phi I}(x, y, z, T), D_{\Phi V}(x, y, z, T), D_{\Phi I S}(x, y, z, T)$ and $D_{\Phi V S}(x, y, z, T)$ are the coefficients of volumetric and surficial diffusions of complexes of radiation defects; $k_{I}(x, y, z, T)$ and $k_{V}(x, y, z, T)$ are the parameters of decay these complexes.

Chemical potential in the Eq.(1) could be determined by the following relation [26]

$$
\mu=E(z)\left[\Omega \sigma_{i j}\left[u_{i j}(x, y, z, t)+u_{j i}(x, y, z, t)\right] / 2,\right.
$$

where $E(z)$ is the Young modulus, $\sigma_{i j}$ is the stress tensor; $u_{i j}=\frac{1}{2}\left(\frac{\partial u_{i}}{\partial x_{j}}+\frac{\partial u_{j}}{\partial x_{i}}\right)$ is the deformation tensor; $u_{i}, u_{j}$ are the components $u_{x}(x, y, z, t), u_{y}(x, y, z, t)$ and $u_{z}(x, y, z, t)$ of the displacement vector $\vec{u}(x, y, z, t) ; x_{i}, x_{j}$ are the coordinate $x, y, z$. The Eq. (3) could be transform to the following form 
Computer Applications: An International Journal (CAIJ), Vol.2, No.1, February 2015

$$
\begin{aligned}
\mu(x, y, z, t)= & {\left[\frac{\partial u_{i}(x, y, z, t)}{\partial x_{j}}+\frac{\partial u_{j}(x, y, z, t)}{\partial x_{i}}\right]\left\{\frac{1}{2}\left[\frac{\partial u_{i}(x, y, z, t)}{\partial x_{j}}+\frac{\partial u_{j}(x, y, z, t)}{\partial x_{i}}\right]-\right.} \\
& \left.-\varepsilon_{0} \delta_{i j}+\frac{\sigma(z) \delta_{i j}}{1-2 \sigma(z)}\left[\frac{\partial u_{k}(x, y, z, t)}{\partial x_{k}}-3 \varepsilon_{0}\right]-K(z) \beta(z)\left[T(x, y, z, t)-T_{0}\right] \delta_{i j}\right) \frac{\Omega}{2} E(z),
\end{aligned}
$$

where $\sigma$ is Poisson coefficient; $\varepsilon_{0}=\left(a_{s}-a_{E L}\right) / a_{E L}$ is the mismatch parameter; $a_{s}, a_{E L}$ are lattice distances of the substrate and the epitaxial layer; $K$ is the modulus of uniform compression; $\beta$ is the thermal expansion coefficient; $T_{r}$ is the equilibrium temperature, which has been assumed as room temperature. Components of displacement vector could be obtained by solution of the following equations [27]

$$
\begin{aligned}
& \rho(z) \frac{\partial^{2} u_{x}(x, y, z, t)}{\partial t^{2}}=\frac{\partial \sigma_{x x}(x, y, z, t)}{\partial x}+\frac{\partial \sigma_{x y}(x, y, z, t)}{\partial y}+\frac{\partial \sigma_{x z}(x, y, z, t)}{\partial z} \\
& \rho(z) \frac{\partial^{2} u_{y}(x, y, z, t)}{\partial t^{2}}=\frac{\partial \sigma_{y x}(x, y, z, t)}{\partial x}+\frac{\partial \sigma_{y y}(x, y, z, t)}{\partial y}+\frac{\partial \sigma_{y z}(x, y, z, t)}{\partial z} \\
& \rho(z) \frac{\partial^{2} u_{z}(x, y, z, t)}{\partial t^{2}}=\frac{\partial \sigma_{z x}(x, y, z, t)}{\partial x}+\frac{\partial \sigma_{z y}(x, y, z, t)}{\partial y}+\frac{\partial \sigma_{z z}(x, y, z, t)}{\partial z},
\end{aligned}
$$

where $\sigma_{i j}=\frac{E(z)}{2[1+\sigma(z)]}\left[\frac{\partial u_{i}(x, y, z, t)}{\partial x_{j}}+\frac{\partial u_{j}(x, y, z, t)}{\partial x_{i}}-\frac{\delta_{i j}}{3} \frac{\partial u_{k}(x, y, z, t)}{\partial x_{k}}\right]+\delta_{i j} \frac{\partial u_{k}(x, y, z, t)}{\partial x_{k}} \times$ $\times K(z)-\beta(z) K(z)\left[T(x, y, z, t)-T_{r}\right], \rho(z)$ is the density of materials of heterostructure, $\delta_{i j}$ Is the Kronecker symbol. With account the relation for $\sigma_{i j}$ last system of equation could be written as

$$
\begin{aligned}
& \rho(z) \frac{\partial^{2} u_{x}(x, y, z, t)}{\partial t^{2}}=\left\{K(z)+\frac{5 E(z)}{6[1+\sigma(z)]}\right\} \frac{\partial^{2} u_{x}(x, y, z, t)}{\partial x^{2}}+\left\{K(z)-\frac{E(z)}{3[1+\sigma(z)]}\right\} \frac{\partial^{2} u_{y}(x, y, z, t)}{\partial x \partial y}+ \\
& +\frac{E(z)}{2[1+\sigma(z)]}\left[\frac{\partial^{2} u_{y}(x, y, z, t)}{\partial y^{2}}+\frac{\partial^{2} u_{z}(x, y, z, t)}{\partial z^{2}}\right]+\left[K(z)+\frac{E(z)}{3[1+\sigma(z)]}\right] \frac{\partial^{2} u_{z}(x, y, z, t)}{\partial x \partial z}- \\
& -K(z) \beta(z) \frac{\partial T(x, y, z, t)}{\partial x} \\
& \rho(z) \frac{\partial^{2} u_{y}(x, y, z, t)}{\partial t^{2}}=\frac{E(z)}{2[1+\sigma(z)]}\left[\frac{\partial^{2} u_{y}(x, y, z, t)}{\partial x^{2}}+\frac{\partial^{2} u_{x}(x, y, z, t)}{\partial x \partial y}\right]-K(z) \beta(z) \frac{\partial T(x, y, z, t)}{\partial y}+ \\
& +\frac{\partial}{\partial z}\left\{\frac{E(z)}{2[1+\sigma(z)]}\left[\frac{\partial u_{y}(x, y, z, t)}{\partial z}+\frac{\partial u_{z}(x, y, z, t)}{\partial y}\right]\right\}+\frac{\partial^{2} u_{y}(x, y, z, t)}{\partial y^{2}}\left\{\frac{5 E(z)}{12[1+\sigma(z)]}+K(z)\right\}+ \\
& +\left\{K(z)-\frac{E(z)}{6[1+\sigma(z)]}\right\} \frac{\partial^{2} u_{y}(x, y, z, t)}{\partial y \partial z}+K(z) \frac{\partial^{2} u_{y}(x, y, z, t)}{\partial x \partial y} \\
& \rho(z) \frac{\partial^{2} u_{z}(x, y, z, t)}{\partial t^{2}}=\frac{E(z)}{2[1+\sigma(z)]}\left[\frac{\partial^{2} u_{z}(x, y, z, t)}{\partial x^{2}}+\frac{\partial^{2} u_{z}(x, y, z, t)}{\partial y^{2}}+\frac{\partial^{2} u_{x}(x, y, z, t)}{\partial x \partial z}+\frac{\partial^{2} u_{y}(x, y, z, t)}{\partial y \partial z}\right]+ \\
& +\frac{\partial}{\partial z}\left\{K(z)\left[\frac{\partial u_{x}(x, y, z, t)}{\partial x}+\frac{\partial u_{y}(x, y, z, t)}{\partial y}+\frac{\partial u_{x}(x, y, z, t)}{\partial z}\right]\right\}-K(z) \beta(z) \frac{\partial T(x, y, z, t)}{\partial z}+
\end{aligned}
$$


Computer Applications: An International Journal (CAIJ), Vol.2, No.1, February 2015

$$
+\frac{1}{6} \frac{\partial}{\partial z}\left\{\frac{E(z)}{1+\sigma(z)}\left[6 \frac{\partial u_{z}(x, y, z, t)}{\partial z}-\frac{\partial u_{x}(x, y, z, t)}{\partial x}-\frac{\partial u_{y}(x, y, z, t)}{\partial y}-\frac{\partial u_{z}(x, y, z, t)}{\partial z}\right]\right\} .
$$

Conditions for the system of Eq. (8) could be written in the form

$$
\begin{aligned}
& \frac{\partial \vec{u}(0, y, z, t)}{\partial x}=0 ; \frac{\partial \vec{u}\left(L_{x}, y, z, t\right)}{\partial x}=0 ; \frac{\partial \vec{u}(x, 0, z, t)}{\partial y}=0 ; \frac{\partial \vec{u}\left(x, L_{y}, z, t\right)}{\partial y}=0 ; \\
& \frac{\partial \vec{u}(x, y, 0, t)}{\partial z}=0 ; \frac{\partial \vec{u}\left(x, y, L_{z}, t\right)}{\partial z}=0 ; \vec{u}(x, y, z, 0)=\vec{u}_{0} ; \vec{u}(x, y, z, \infty)=\vec{u}_{0} .
\end{aligned}
$$

We determine spatio-temporal distributions of concentrations of dopant and radiati-on defects by solving the Eqs.(1), (3) and (5) framework standard method of averaging of function corrections [25,31]. Previously we transform the Eqs.(1), (3) and (5) to the following form with account initial distributions of the considered concentrations

$$
\begin{aligned}
& \frac{\partial C(x, y, z, t)}{\partial t}=\frac{\partial}{\partial x}\left[D \frac{\partial C(x, y, z, t)}{\partial x}\right]+\frac{\partial}{\partial y}\left[D \frac{\partial C(x, y, z, t)}{\partial y}\right]+\frac{\partial}{\partial z}\left[D \frac{\partial C(x, y, z, t)}{\partial z}\right]+ \\
& +f_{C}(x, y, z) \delta(t)+\Omega \frac{\partial}{\partial x}\left[\frac{D_{S}}{k T} \nabla_{S} \mu(x, y, z, t) \int_{0}^{L_{\tilde{z}}} C(x, y, W, t) d W\right]+ \\
& +\Omega \frac{\partial}{\partial y}\left[\frac{D_{S}}{k T} \nabla_{S} \mu(x, y, z, t) \int_{0}^{L_{z}} C(x, y, W, t) d W\right] \\
& \frac{\partial I(x, y, z, t)}{\partial t}=\frac{\partial}{\partial x}\left[D_{I}(x, y, z, T) \frac{\partial I(x, y, z, t)}{\partial x}\right]+\frac{\partial}{\partial y}\left[D_{I}(x, y, z, T) \frac{\partial I(x, y, z, t)}{\partial y}\right]+ \\
& +\frac{\partial}{\partial z}\left[D_{I}(x, y, z, T) \frac{\partial I(x, y, z, t)}{\partial z}\right]-k_{I, V}(x, y, z, T) I(x, y, z, t) V(x, y, z, t)+f_{I}(x, y, z) \delta(t)- \\
& -k_{I, I}(x, y, z, T) I^{2}(x, y, z, t)+\Omega \frac{\partial}{\partial x}\left[\frac{D_{I S}}{k T} \nabla_{S} \mu(x, y, z, t) \int_{0}^{L_{z}} I(x, y, W, t) d W\right]+ \\
& +\Omega \frac{\partial}{\partial y}\left[\frac{D_{I S}}{k T} \nabla_{s} \mu(x, y, z, t) \int_{0}^{L_{z}} I(x, y, W, t) d W\right] \\
& \frac{\partial V(x, y, z, t)}{\partial t}=\frac{\partial}{\partial x}\left[D_{V}(x, y, z, T) \frac{\partial V(x, y, z, t)}{\partial x}\right]+\frac{\partial}{\partial y}\left[D_{V}(x, y, z, T) \frac{\partial V(x, y, z, t)}{\partial y}\right]+ \\
& +\frac{\partial}{\partial z}\left[D_{V}(x, y, z, T) \frac{\partial V(x, y, z, t)}{\partial z}\right]-k_{I, V}(x, y, z, T) I(x, y, z, t) V(x, y, z, t)+f_{V}(x, y, z) \delta(t)- \\
& -k_{V, V}(x, y, z, T) V^{2}(x, y, z, t)+\Omega \frac{\partial}{\partial x}\left[\frac{D_{V S}}{k T} \nabla_{s} \mu(x, y, z, t) \int_{0}^{L_{z}} V(x, y, W, t) d W\right]+ \\
& +\Omega \frac{\partial}{\partial y}\left[\frac{D_{V S}}{k T} \nabla_{s} \mu(x, y, z, t) \int_{0}^{L_{z}} V(x, y, W, t) d W\right] \\
& \frac{\partial \Phi_{I}(x, y, z, t)}{\partial t}=\frac{\partial}{\partial x}\left[D_{\Phi I}(x, y, z, T) \frac{\partial \Phi_{I}(x, y, z, t)}{\partial x}\right]+\frac{\partial}{\partial y}\left[D_{\Phi I}(x, y, z, T) \frac{\partial \Phi_{I}(x, y, z, t)}{\partial y}\right]+ \\
& +\frac{\partial}{\partial z}\left[D_{\Phi_{I}}(x, y, z, T) \frac{\partial \Phi_{I}(x, y, z, t)}{\partial z}\right]+\Omega \frac{\partial}{\partial x}\left[\frac{D_{\Phi_{I} S}}{k T} \nabla_{S} \mu(x, y, z, t) \int_{0}^{L_{z}} \Phi_{I}(x, y, W, t) d W\right]+
\end{aligned}
$$


Computer Applications: An International Journal (CAIJ), Vol.2, No.1, February 2015

$$
\begin{gathered}
+\Omega \frac{\partial}{\partial y}\left[\frac{D_{\Phi_{I} S}}{k T} \nabla_{S} \mu(x, y, z, t) \int_{0}^{L_{2}} \Phi_{I}(x, y, W, t) d W\right]+k_{I}(x, y, z, T) I(x, y, z, t)+ \\
+k_{I, I}(x, y, z, T) I^{2}(x, y, z, t)+f_{\Phi_{I}}(x, y, z) \delta(t) \\
\frac{\partial \Phi_{V}(x, y, z, t)}{\partial t}=\frac{\partial}{\partial x}\left[D_{\Phi V}(x, y, z, T) \frac{\partial \Phi_{V}(x, y, z, t)}{\partial x}\right]+\frac{\partial}{\partial y}\left[D_{\Phi V}(x, y, z, T) \frac{\partial \Phi_{V}(x, y, z, t)}{\partial y}\right]+ \\
+\frac{\partial}{\partial z}\left[D_{\Phi_{V}}(x, y, z, T) \frac{\partial \Phi_{V}(x, y, z, t)}{\partial z}\right]+\Omega \frac{\partial}{\partial x}\left[\frac{D_{\Phi_{V} S}}{k T} \nabla_{S} \mu(x, y, z, t) \int_{0}^{L_{z}} \Phi_{V}(x, y, W, t) d W\right]+ \\
+\Omega \frac{\partial}{\partial y}\left[\frac{D_{\Phi_{V} S}}{k T} \nabla_{S} \mu(x, y, z, t) \int_{0}^{L_{2}} \Phi_{V}(x, y, W, t) d W\right]+k_{V}(x, y, z, T) V(x, y, z, t)+ \\
+k_{V, V}(x, y, z, T) V^{2}(x, y, z, t)+f_{\Phi_{V}}(x, y, z) \delta(t) .
\end{gathered}
$$

Farther we replace the required functions in right sides of Eqs. (1a), (3a) and (5a) on their not yet known average values $\alpha_{1 \rho}$. In this situation we obtain equations for the first-order approximations of the considered concentrations in the following form

$$
\begin{gathered}
\frac{\partial C_{1}(x, y, z, t)}{\partial t}=\alpha_{1 C} \Omega \frac{\partial}{\partial x}\left[z \frac{D_{S}}{k T} \nabla_{S} \mu(x, y, z, t)\right]+\alpha_{1 C} \Omega \frac{\partial}{\partial y}\left[z \frac{D_{S}}{k T} \nabla_{S} \mu(x, y, z, t)\right]+ \\
+f_{C}(x, y, z) \delta(t) \\
\left\{\begin{array}{l}
\frac{\partial I_{1}(x, y, z, t)}{\partial t}=f_{I}(x, y, z) \delta(t)+\alpha_{1 I} z \Omega \frac{\partial}{\partial x}\left[\frac{D_{I S}}{k T} \nabla_{S} \mu(x, y, z, t)\right]+ \\
+\alpha_{1 I} \Omega \frac{\partial}{\partial y}\left[z \frac{D_{I S}}{k T} \nabla_{S} \mu(x, y, z, t)\right]-\alpha_{1 I}^{2} k k_{I, I}(x, y, z, T)-\alpha_{1 I} \alpha_{1 V} k_{I, V}(x, y, z, T) \\
\frac{\partial V_{1}(x, y, z, t)}{\partial t}=f_{V}(x, y, z) \delta(t)+\alpha_{1 V} z \Omega \frac{\partial}{\partial x}\left[\frac{D_{V S}}{k T} \nabla_{S} \mu(x, y, z, t)\right]+ \\
+\alpha_{1 V} \Omega \frac{\partial}{\partial y}\left[z \frac{D_{V S}}{k T} \nabla_{S} \mu(x, y, z, t)\right]-\alpha_{1 V}^{2} k_{V, V}(x, y, z, T)-\alpha_{1 I} \alpha_{1 V} k_{I, V}(x, y, z, T) \\
\frac{\partial \Phi_{1 I}(x, y, z, t)}{\partial t}=f_{\Phi_{I}}(x, y, z) \delta(t)+\alpha_{1 \Phi_{I}} z \Omega \frac{\partial}{\partial x}\left[\frac{D_{\Phi_{I} S}}{k T} \nabla_{s} \mu(x, y, z, t)\right]+k_{I}(x, y, z, T) \times \\
\times I(x, y, z, t)+\alpha_{1 \Phi_{I}} z \Omega \frac{\partial}{\partial y}\left[\frac{D_{\Phi_{I} S}}{k T} \nabla_{S} \mu(x, y, z, t)\right]+k_{I, I}(x, y, z, T) I^{2}(x, y, z, t) \\
\frac{\partial \Phi_{1 V}(x, y, z, t)}{\partial t}=f_{\Phi_{V}}(x, y, z) \delta(t)+\alpha_{1 \Phi_{V}} z \Omega \frac{\partial}{\partial x}\left[\frac{D_{\Phi_{V} S}}{k T} \nabla_{S} \mu(x, y, z, t)\right]+k_{V}(x, y, z, T) \times \\
\times V(x, y, z, t)+\alpha_{1 \Phi_{V}} z \Omega \frac{\partial}{\partial y}\left[\frac{D_{\Phi_{V} S}}{k T} \nabla_{S} \mu(x, y, z, t)\right]+k_{V, V}(x, y, z, T) V^{2}(x, y, z, t)
\end{array}\right.
\end{gathered}
$$

Integration of the left and right sides of Eqs. $(1 b),(3 b)$ and $(5 b)$ gives us possibility to obtain relations for above first-order approximations in the following form 
Computer Applications: An International Journal (CAIJ), Vol.2, No.1, February 2015

$$
\begin{aligned}
& C_{1}(x, y, z, t)=\alpha_{1 C} \Omega \frac{\partial}{\partial x} \int_{0}^{t} D_{S L}(x, y, z, T) \nabla_{s} \mu(x, y, z, \tau)\left[1+\varsigma_{1} \frac{V(x, y, z, \tau)}{V^{*}}+\varsigma_{2} \frac{V^{2}(x, y, z, \tau)}{\left(V^{*}\right)^{2}}\right] \times \\
& \left.\times \frac{z}{k T}\left[1+\frac{\xi_{S} \alpha_{1 C}^{\gamma}}{P^{\gamma}(x, y, z, T)}\right] d \tau\right\}+\alpha_{1 C} \frac{\partial}{\partial y} \int_{0}^{t} D_{S L}(x, y, z, T)\left[1+\frac{\xi_{S} \alpha_{1 C}^{\gamma}}{P^{\gamma}(x, y, z, T)}\right]\left[1+\frac{\xi_{S} \alpha_{1 C}^{\gamma}}{P^{\gamma}(x, y, z, T)}\right] \times \\
& \times z \frac{\Omega}{k T}\left[1+\varsigma_{1} \frac{V(x, y, z, \tau)}{V^{*}}+\varsigma_{2} \frac{V^{2}(x, y, z, \tau)}{\left(V^{*}\right)^{2}}\right] d \tau+f_{C}(x, y, z) \\
& \left\{\begin{array}{l}
I_{1}(x, y, z, t)=f_{I}(x, y, z)-\alpha_{1 I}^{2} \int_{0}^{t} k_{I, I}(x, y, z, T) d \tau-\alpha_{1 I} \alpha_{1 V} \int_{0}^{t} k_{I, V}(x, y, z, T) d \tau \\
+\alpha_{1 I} z \Omega \frac{\partial}{\partial x} \int_{0}^{t} \frac{D_{I S}}{k T} \nabla_{s} \mu(x, y, z, \tau) d \tau+\alpha_{1 I} z \Omega \frac{\partial}{\partial y} \int_{0}^{t} \frac{D_{I S}}{k T} \nabla_{S} \mu(x, y, z, \tau) d \tau \\
V_{1}(x, y, z, t)=f_{V}(x, y, z)-\alpha_{1 V}^{2} \int_{0}^{t} k_{V, V}(x, y, z, T) d \tau-\alpha_{1 I} \alpha_{1 V} \int_{0}^{t} k_{I, V}(x, y, z, T) d \tau+ \\
+\alpha_{1 V} z \Omega \frac{\partial}{\partial x} \int_{0}^{t} \frac{D_{I S}}{k T} \nabla_{S} \mu(x, y, z, \tau) d \tau+\alpha_{1 V} z \Omega \frac{\partial}{\partial y} \int_{0}^{t} \frac{D_{I S}}{k T} \nabla_{S} \mu(x, y, z, \tau) d \tau
\end{array}\right. \\
& \left\{\begin{array}{l}
\Phi_{1 I}(x, y, z, t)=f_{\Phi_{I}}(x, y, z)+\alpha_{1 \Phi_{I}} z \Omega \frac{\partial}{\partial x} \int_{0}^{t} \frac{D_{\Phi_{I} S}}{k T} \nabla_{S} \mu(x, y, z, \tau) d \tau+ \\
+\alpha_{1 \Phi_{I}} z \Omega \frac{\partial}{\partial y} \int_{0}^{t} \frac{D_{\Phi_{I} S}}{k T} \nabla_{S} \mu(x, y, z, \tau) d \tau+\int_{0}^{t} k_{I}(x, y, z, T) I(x, y, z, \tau) d \tau+
\end{array}\right. \\
& \left\{+\int_{0}^{t} k_{I, I}(x, y, z, T) I^{2}(x, y, z, \tau) d \tau\right. \\
& \Phi_{1 V}(x, y, z, t)=f_{\Phi_{V}}(x, y, z)+\alpha_{1 \Phi_{V}} z \Omega \frac{\partial}{\partial x} \int_{0}^{t} \frac{D_{\Phi_{V} S}}{k T} \nabla_{S} \mu(x, y, z, \tau) d \tau+ \\
& \begin{array}{l}
+\alpha_{1 \Phi_{V}} z \Omega \frac{\partial}{\partial y} \int_{0}^{t} \frac{D_{\Phi_{V} S}}{k T} \nabla_{S} \mu(x, y, z, \tau) d \tau+\int_{0}^{t} k_{V}(x, y, z, T) V(x, y, z, \tau) d \tau+ \\
+\int_{0}^{t} k_{V, V}(x, y, z, T) V^{2}(x, y, z, \tau) d \tau
\end{array}
\end{aligned}
$$

We determine average values of the first-order approximations of required functions by using the following relations $[25,31]$

$$
\alpha_{1 \rho}=\frac{1}{\Theta L_{x} L_{y} L_{z}} \int_{0}^{\Theta} \int_{0}^{L_{x}} \int_{0}^{L_{y} L_{z}} \int_{0} \rho_{1}(x, y, z, t) d z d y d x d t
$$

Substitution of these relations (1c), (3c) and (5c) into relation (9) gives us possibility to obtain the following relations

$$
\begin{gathered}
\alpha_{1 C}=\frac{1}{L_{x} L_{y} L_{z}} \int_{0}^{L_{x} L_{y} L_{z}} \int_{0} f_{C}(x, y, z) d z d y d x \\
\alpha_{1 I}=\sqrt{\frac{\left(a_{3}+A\right)^{2}}{4 a_{4}^{2}}-4\left(B+\frac{\Theta a_{3} B+\Theta^{2} L_{x} L_{y} L_{z} a_{1}}{a_{4}}\right)}-\frac{a_{3}+A}{4 a_{4}},
\end{gathered}
$$


Computer Applications: An International Journal (CAIJ), Vol.2, No.1, February 2015

$$
\alpha_{1 V}=\frac{1}{S_{I V 00}}\left[\frac{\Theta}{\alpha_{1 I}} \int_{0}^{L_{x}} \int_{0}^{L_{2} L_{z}} \int_{0} f_{I}(x, y, z) d z d y d x-\alpha_{1 I} S_{I I 00}-\Theta L_{x} L_{y} L_{z}\right],
$$

where $S_{\rho \rho i j}=\int_{0}^{\Theta}(\Theta-t) \int_{0}^{L_{L}} \int_{0}^{L_{L} L_{z}} \int_{0}^{k_{\rho, \rho}} k_{\rho}(x, y, z, T) I_{1}^{i}(x, y, z, t) V_{1}^{j}(x, y, z, t) d z d y d x d t, a_{4}=S_{I I 00} \times$

$$
\begin{aligned}
& \times\left(S_{I V 00}^{2}-S_{I I 00} S_{V V 00}\right), a_{3}=S_{I V 00} S_{I I 00}+S_{I V 00}^{2}-S_{I I 00} S_{V V 00}, a_{2}=\int_{0}^{L_{L}} \int_{0}^{L_{y} L_{2}} \int_{0} f_{V}(x, y, z) d z d y d x \times \\
& \times S_{I V 00} S_{I V 00}^{2}+2 S_{V V 00} S_{I I 00} \int_{0}^{L_{x}} \int_{0}^{L_{y}} \int_{0}^{L_{z}} f_{I}(x, y, z) d z d y d x-S_{I V 00}^{2} \int_{0}^{L_{x}} \int_{0}^{L_{y}} \int_{0}^{L_{z}} f_{I}(x, y, z) d z d y d x- \\
& -\Theta L_{x}^{2} L_{y}^{2} L_{z}^{2} S_{V V 00}+S_{I V 00} \Theta L_{x}^{2} L_{y}^{2} L_{z}^{2}, a_{1}=S_{I V 00} \int_{0}^{L_{x} L_{y} L_{z}} \int_{0}^{0} f_{I}(x, y, z) d z d y d x, A=\sqrt{8 y+\Theta^{2} \frac{a_{3}^{2}}{a_{4}^{2}}-4 \Theta \frac{a_{2}}{a_{4}}}, \\
& a_{0}=S_{V V 00}\left[\int_{0}^{L_{x}} \int_{0}^{L_{y} L_{2}} \int_{0}^{2} f_{I}(x, y, z) d z d y d x\right]^{2}, B=\frac{\Theta a_{2}}{6 a_{4}}+\sqrt[3]{\sqrt{q^{2}+p^{3}}-q}-\sqrt[3]{\sqrt{q^{2}+p^{3}}+q}, \\
& q=\frac{\Theta^{3} a_{2}}{24 a_{4}^{2}}\left(4 a_{0}-\Theta L_{x} L_{y} L_{z} \frac{a_{1} a_{3}}{a_{4}}\right)-\Theta^{2} \frac{a_{0}}{8 a_{4}^{2}}\left(4 \Theta a_{2}-\Theta^{2} \frac{a_{3}^{2}}{a_{4}}\right)-\frac{\Theta^{3} a_{2}^{3}}{54 a_{4}^{3}}-L_{x}^{2} L_{y}^{2} L_{z}^{2} \frac{\Theta^{4} a_{1}^{2}}{8 a_{4}^{2}}, \\
& p=\Theta^{2} \frac{4 a_{0} a_{4}-\Theta L_{x} L_{y} L_{z} a_{1} a_{3}}{12 a_{4}^{2}}-\frac{\Theta a_{2}}{18 a_{4}}, \\
& \alpha_{1 \Phi_{I}}=\frac{R_{I 1}}{\Theta L_{x} L_{y} L_{z}}+\frac{S_{I I 20}}{\Theta L_{x} L_{y} L_{z}}+\frac{1}{L_{x} L_{y} L_{z}} \int_{0}^{L_{x}} \int_{0}^{L_{y} L_{z}} \int_{0} f_{\Phi_{I}}(x, y, z) d z d y d x \\
& \alpha_{1 \Phi_{V}}=\frac{R_{V 1}}{\Theta L_{x} L_{y} L_{z}}+\frac{S_{V V 20}}{\Theta L_{x} L_{y} L_{z}}+\frac{1}{L_{x} L_{y} L_{z}} \int_{0}^{L_{x} L_{y} L_{z}} \int_{0} \int_{0} f_{\Phi_{V}}(x, y, z) d z d y d x
\end{aligned}
$$

where $R_{\rho i}=\int_{0}^{\Theta}(\Theta-t) \iint_{0}^{L_{L}} \int_{0}^{L_{y}} \int_{0}^{L_{z}} k_{I}(x, y, z, T) I_{1}^{i}(x, y, z, t) d z d y d x d t$.

We obtain the second-order approximations and approximations with higher orders of concentrations of dopant and radiation defects by using standard iteration procedure of method of averaging of function corrections [25,31]. Framework this procedure to calculate $n$-th-order approximations of concentrations of dopant and radiation defects we replace the required concentrations $C(x, y, z, t), I(x, y, z, t), V(x, y, z, t), \Phi_{I}(x, y, z, t)$ and $\Phi_{V}(x, y, z, t)$ in the right sides of Eqs. (1a), (3a), (5a) on the following sums $\alpha_{n}+\rho_{n-1}(x, y, z, t)$. This substitution gives us possibility to obtain equations for the second-order approximations of the required concentrations

$$
\begin{aligned}
& \quad \frac{\partial C_{2}(x, y, z, t)}{\partial t}=\frac{\partial}{\partial x}\left(\left\{1+\xi \frac{\left[\alpha_{2 C}+C_{1}(x, y, z, t)\right]^{\gamma}}{P^{\gamma}(x, y, z, T)}\right\}\left[1+\varsigma_{1} \frac{V(x, y, z, t)}{V^{*}}+\varsigma_{2} \frac{V^{2}(x, y, z, t)}{\left.V^{*}\right)^{2}}\right] \times\right. \\
& \left.\times D_{L}(x, y, z, T) \frac{\partial C_{1}(x, y, z, t)}{\partial x}\right)+\frac{\partial}{\partial y}\left(\left[1+\varsigma_{1} \frac{V(x, y, z, t)}{V^{*}}+\varsigma_{2} \frac{V^{2}(x, y, z, t)}{\left(V^{*}\right)^{2}}\right] \frac{\partial C_{1}(x, y, z, t)}{\partial y} \times\right. \\
& \left.\times D_{L}(x, y, z, T)\left\{1+\xi \frac{\left[\alpha_{2 C}+C_{1}(x, y, z, t)\right]^{\gamma}}{P^{\gamma}(x, y, z, T)}\right\}\right)+\frac{\partial}{\partial z}\left(\left[1+\varsigma_{1} \frac{V(x, y, z, t)}{V^{*}}+\varsigma_{2} \frac{V^{2}(x, y, z, t)}{\left(V^{*}\right)^{2}}\right] \times\right. \\
& \left.\quad \times D_{L}(x, y, z, T) \frac{\partial C_{1}(x, y, z, t)}{\partial z}\left\{1+\xi \frac{\left[\alpha_{2 C}+C_{1}(x, y, z, t)\right]^{\gamma}}{P^{\gamma}(x, y, z, T)}\right\}\right)+f_{C}(x, y, z) \delta(t)+\frac{\partial}{\partial x}\left[\frac{D_{s}}{k T} \times\right.
\end{aligned}
$$


Computer Applications: An International Journal (CAIJ), Vol.2, No.1, February 2015

$$
\begin{aligned}
& \left.\times \nabla_{S} \mu(x, y, z, t) \int_{0}^{L_{\tilde{z}}} C(x, y, W, t) d W\right] \Omega+\Omega \frac{\partial}{\partial y}\left[\frac{D_{s}}{k T} \nabla_{s} \mu(x, y, z, t) \int_{0}^{L_{z}} C(x, y, W, t) d W\right] \\
& \frac{\partial I_{2}(x, y, z, t)}{\partial t}=\frac{\partial}{\partial x}\left[D_{I}(x, y, z, T) \frac{\partial I_{1}(x, y, z, t)}{\partial x}\right]+\frac{\partial}{\partial y}\left[D_{I}(x, y, z, T) \frac{\partial I_{1}(x, y, z, t)}{\partial y}\right]- \\
& +\frac{\partial}{\partial z}\left[D_{I}(x, y, z, T) \frac{\partial I_{1}(x, y, z, t)}{\partial z}\right]-k_{I, V}(x, y, z, T)\left[\alpha_{1 I}+I_{1}(x, y, z, t)\right]\left[\alpha_{1 V}+V_{1}(x, y, z, t)\right]- \\
& -k_{I, I}(x, y, z, T)\left[\alpha_{1 I}+I_{1}(x, y, z, t)\right]^{2}+\Omega \frac{\partial}{\partial x}\left\{\frac{D_{I S}}{k T} \nabla_{S} \mu(x, y, z, t) \int_{0}^{L_{L}}\left[\alpha_{2 I}+I_{1}(x, y, W, t)\right] d W\right\}+ \\
& +\Omega \frac{\partial}{\partial y}\left\{\frac{D_{I S}}{k T} \nabla_{S} \mu(x, y, z, t) \int_{0}^{L_{\tilde{z}}}\left[\alpha_{2 I}+I_{1}(x, y, W, t)\right] d W\right\}(3 d) \\
& \frac{\partial V_{2}(x, y, z, t)}{\partial t}=\frac{\partial}{\partial x}\left[D_{V}(x, y, z, T) \frac{\partial V_{1}(x, y, z, t)}{\partial x}\right]+\frac{\partial}{\partial y}\left[D_{V}(x, y, z, T) \frac{\partial V_{1}(x, y, z, t)}{\partial y}\right]- \\
& +\frac{\partial}{\partial z}\left[D_{V}(x, y, z, T) \frac{\partial V_{1}(x, y, z, t)}{\partial z}\right]-k_{I, V}(x, y, z, T)\left[\alpha_{1 I}+I_{1}(x, y, z, t)\right]\left[\alpha_{1 V}+V_{1}(x, y, z, t)\right]- \\
& -k_{V, V}(x, y, z, T)\left[\alpha_{1 V}+V_{1}(x, y, z, t)\right]^{2}+\Omega \frac{\partial}{\partial x}\left\{\frac{D_{V S}}{k T} \nabla_{S} \mu(x, y, z, t) \int_{0}^{L_{L}}\left[\alpha_{2 V}+V_{1}(x, y, W, t)\right] d W\right\}+ \\
& +\Omega \frac{\partial}{\partial y}\left\{\frac{D_{V S}}{k T} \nabla_{S} \mu(x, y, z, t) \int_{0}^{L_{z}}\left[\alpha_{2 V}+V_{1}(x, y, W, t)\right] d W\right\} \\
& \frac{\partial \Phi_{2 I}(x, y, z, t)}{\partial t}=\frac{\partial}{\partial x}\left[D_{\Phi_{I}}(x, y, z, T) \frac{\partial \Phi_{1 I}(x, y, z, t)}{\partial x}\right]+\frac{\partial}{\partial y}\left[D_{\Phi_{I}}(x, y, z, T) \frac{\partial \Phi_{1 I}(x, y, z, t)}{\partial y}\right]+ \\
& +k_{I, I}(x, y, z, T) I^{2}(x, y, z, t)+\Omega \frac{\partial}{\partial x}\left\{\frac{D_{\Phi_{I} S}}{k T} \nabla_{S} \mu(x, y, z, t) \int_{0}^{L_{z}}\left[\alpha_{2 \Phi_{I}}+\Phi_{1 I}(x, y, W, t)\right] d W\right\}+ \\
& +\Omega \frac{\partial}{\partial y}\left\{\frac{D_{\Phi_{I} S}}{k T} \nabla_{S} \mu(x, y, z, t) \int_{0}^{L_{2}}\left[\alpha_{2 \Phi_{I}}+\Phi_{1 I}(x, y, W, t)\right] d W\right\}+k_{I}(x, y, z, T) I(x, y, z, t)+ \\
& +\frac{\partial}{\partial z}\left[D_{\Phi_{I}}(x, y, z, T) \frac{\partial \Phi_{1 I}(x, y, z, t)}{\partial z}\right]+f_{\Phi_{I}}(x, y, z) \delta(t) \\
& \frac{\partial \Phi_{2 V}(x, y, z, t)}{\partial t}=\frac{\partial}{\partial x}\left[D_{\Phi_{V}}(x, y, z, T) \frac{\partial \Phi_{1 V}(x, y, z, t)}{\partial x}\right]+\frac{\partial}{\partial y}\left[D_{\Phi_{V}}(x, y, z, T) \frac{\partial \Phi_{1 V}(x, y, z, t)}{\partial y}\right]+ \\
& +k_{V, V}(x, y, z, T) V^{2}(x, y, z, t)+\Omega \frac{\partial}{\partial x}\left\{\frac{D_{\Phi_{V} S}}{k T} \nabla_{S} \mu(x, y, z, t) \int_{0}^{L_{\varepsilon}}\left[\alpha_{2 \Phi_{V}}+\Phi_{1 V}(x, y, W, t)\right] d W\right\}+ \\
& +\Omega \frac{\partial}{\partial y}\left\{\frac{D_{\Phi_{V} S}}{k T} \nabla_{S} \mu(x, y, z, t) \int_{0}^{L_{z}}\left[\alpha_{2 \Phi_{V}}+\Phi_{1 V}(x, y, W, t)\right] d W\right\}+k_{V}(x, y, z, T) V(x, y, z, t)+ \\
& +\frac{\partial}{\partial z}\left[D_{\Phi_{V}}(x, y, z, T) \frac{\partial \Phi_{1 V}(x, y, z, t)}{\partial z}\right]+f_{\Phi_{V}}(x, y, z) \delta(t) .
\end{aligned}
$$

Integration of left and right sides of Eqs. $(1 d),(3 d)$ and $(5 d)$ gives us possibility to obtain final relations for the second-order approximations of the required concentrations of dopant and radiation defects in the following form 
Computer Applications: An International Journal (CAIJ), Vol.2, No.1, February 2015

$$
\begin{aligned}
& C_{2}(x, y, z, t)=\frac{\partial}{\partial x} \int_{0}^{t}\left\{1+\xi \frac{\left[\alpha_{2 C}+C_{1}(x, y, z, \tau)\right]^{\gamma}}{P^{\gamma}(x, y, z, T)}\right\}\left[1+\varsigma_{1} \frac{V(x, y, z, \tau)}{V^{*}}+\varsigma_{2} \frac{V^{2}(x, y, z, \tau)}{\left(V^{*}\right)^{2}}\right] \times \\
& \times D_{L}(x, y, z, T) \frac{\partial C_{1}(x, y, z, \tau)}{\partial x} d \tau+\frac{\partial}{\partial y} \int_{0}^{t} D_{L}(x, y, z, T)\left[1+\varsigma_{1} \frac{V(x, y, z, \tau)}{V^{*}}+\varsigma_{2} \frac{V^{2}(x, y, z, \tau)}{\left(V^{*}\right)^{2}}\right] \times \\
& \times \frac{\partial C_{1}(x, y, z, \tau)}{\partial y}\left\{1+\xi \frac{\left[\alpha_{2 C}+C_{1}(x, y, z, t)\right]^{\gamma}}{P^{\gamma}(x, y, z, T)}\right\}+\frac{\partial}{\partial z} \int_{0}^{t}\left[1+\varsigma_{1} \frac{V(x, y, z, \tau)}{V^{*}}+\varsigma_{2} \frac{V^{2}(x, y, z, \tau)}{\left(V^{*}\right)^{2}}\right] \times \\
& \times D_{L}(x, y, z, T) \frac{\partial C_{1}(x, y, z, \tau)}{\partial z}\left\{1+\xi \frac{\left[\alpha_{2 C}+C_{1}(x, y, z, \tau)\right]^{\gamma}}{P^{\gamma}(x, y, z, T)}\right\} d \tau+f_{C}(x, y, z)+\Omega \frac{\partial}{\partial x} \int_{0}^{t} \frac{D_{S}}{k T} \times \\
& \times \nabla_{S} \mu(x, y, z, \tau) \int_{0}^{L_{z}}\left[\alpha_{2 C}+C_{1}(x, y, W, \tau)\right] d W d \tau+\Omega \frac{\partial}{\partial y} \int_{0}^{t} \frac{D_{S}}{k T} \int_{0}^{L_{z}}\left[\alpha_{2 C}+C_{1}(x, y, W, \tau)\right] d W \times \\
& \times \nabla_{s} \mu(x, y, z, \tau) d \tau \\
& I_{2}(x, y, z, t)=\frac{\partial}{\partial x} \int_{0}^{t} D_{I}(x, y, z, T) \frac{\partial I_{1}(x, y, z, \tau)}{\partial x} d \tau+\frac{\partial}{\partial y} \int_{0}^{t} D_{I}(x, y, z, T) \frac{\partial I_{1}(x, y, z, \tau)}{\partial y} d \tau+ \\
& +\frac{\partial}{\partial z} \int_{0}^{t} D_{I}(x, y, z, T) \frac{\partial I_{1}(x, y, z, \tau)}{\partial z} d \tau-\int_{0}^{t} k_{I, I}(x, y, z, T)\left[\alpha_{2 I}+I_{1}(x, y, z, \tau)\right]^{2} d \tau- \\
& +\Omega \frac{\partial}{\partial x} \int_{0}^{t} \nabla_{S} \mu(x, y, z, \tau) \frac{D_{I S}}{k T} \int_{0}^{L_{\varepsilon}}\left[\alpha_{2 I}+I_{1}(x, y, W, \tau)\right] d W d \tau+\Omega \frac{\partial}{\partial y} \int_{0}^{t} \frac{D_{I S}}{k T} \nabla_{S} \mu(x, y, z, \tau) \times \\
& \times \int_{0}^{L_{z}}\left[\alpha_{2 I}+I_{1}(x, y, W, \tau)\right] d W d \tau-\int_{0}^{t} k_{I, V}(x, y, z, T)\left[\alpha_{2 I}+I_{1}(x, y, z, \tau)\right]\left[\alpha_{2 V}+V_{1}(x, y, z, \tau)\right] d \tau+ \\
& +f_{I}(x, y, z) \\
& V_{2}(x, y, z, t)=\frac{\partial}{\partial x} \int_{0}^{t} D_{V}(x, y, z, T) \frac{\partial V_{1}(x, y, z, \tau)}{\partial x} d \tau+\frac{\partial}{\partial y_{0}} \int_{0}^{t} D_{V}(x, y, z, T) \frac{\partial V_{1}(x, y, z, \tau)}{\partial y} d \tau+ \\
& +\frac{\partial}{\partial z} \int_{0}^{t} D_{V}(x, y, z, T) \frac{\partial V_{1}(x, y, z, \tau)}{\partial z} d \tau-\int_{0}^{t} k_{V, V}(x, y, z, T)\left[\alpha_{2 V}+V_{1}(x, y, z, \tau)\right]^{2} d \tau- \\
& +\Omega \frac{\partial}{\partial x} \int_{0}^{t} \nabla_{S} \mu(x, y, z, \tau) \frac{D_{V S}}{k T} \int_{0}^{L_{\varepsilon}}\left[\alpha_{2 V}+V_{1}(x, y, W, \tau)\right] d W d \tau+\Omega \frac{\partial}{\partial y} \int_{0}^{t} \frac{D_{V S}}{k T} \nabla_{s} \mu(x, y, z, \tau) \times \\
& \times \int_{0}^{L_{z}}\left[\alpha_{2 V}+V_{1}(x, y, W, \tau)\right] d W d \tau-\int_{0}^{t} k_{I, V}(x, y, z, T)\left[\alpha_{2 I}+I_{1}(x, y, z, \tau)\right]\left[\alpha_{2 V}+V_{1}(x, y, z, \tau)\right] d \tau+ \\
& +f_{V}(x, y, z) \\
& \Phi_{2 I}(x, y, z, t)=\frac{\partial}{\partial x} \int_{0}^{t} D_{\Phi_{I}}(x, y, z, T) \frac{\partial \Phi_{1 I}(x, y, z, \tau)}{\partial x} d \tau+\frac{\partial}{\partial y} \int_{0}^{t} \frac{\partial \Phi_{1 I}(x, y, z, \tau)}{\partial y} \times \\
& \times D_{\Phi_{I}}(x, y, z, T) d \tau+\frac{\partial}{\partial z} \int_{0}^{t} D_{\Phi_{I}}(x, y, z, T) \frac{\partial \Phi_{1 I}(x, y, z, \tau)}{\partial z} d \tau+\Omega \frac{\partial}{\partial x} \int_{0}^{t} \frac{D_{\Phi_{I} S}}{k T} \nabla_{S} \mu(x, y, z, \tau) \times \\
& \times \int_{0}^{L_{\tau}}\left[\alpha_{2 \Phi_{I}}+\Phi_{1 I}(x, y, W, \tau)\right] d W d \tau+\Omega \frac{\partial}{\partial y} \int_{0}^{t} \nabla_{S} \mu(x, y, z, \tau) \int_{0}^{L_{2}}\left[\alpha_{2 \Phi_{I}}+\Phi_{1 I}(x, y, W, \tau)\right] d W \times \\
& \times \frac{D_{\Phi_{I} S}}{k T} d \tau+\int_{0}^{t} k_{I, I}(x, y, z, T) I^{2}(x, y, z, \tau) d \tau+\int_{0}^{t} k_{I}(x, y, z, T) I(x, y, z, \tau) d \tau+f_{\Phi_{I}}(x, y, z) \\
& \Phi_{2 V}(x, y, z, t)=\frac{\partial}{\partial x} \int_{0}^{t} D_{\Phi_{V}}(x, y, z, T) \frac{\partial \Phi_{1 V}(x, y, z, \tau)}{\partial x} d \tau+\frac{\partial}{\partial y} \int_{0}^{t} \frac{\partial \Phi_{1 V}(x, y, z, \tau)}{\partial y} \times
\end{aligned}
$$


Computer Applications: An International Journal (CAIJ), Vol.2, No.1, February 2015

$$
\begin{aligned}
& \times D_{\Phi_{V}}(x, y, z, T) d \tau+\frac{\partial}{\partial z} \int_{0}^{t} D_{\Phi_{V}}(x, y, z, T) \frac{\partial \Phi_{1 V}(x, y, z, \tau)}{\partial z} d \tau+\Omega \frac{\partial}{\partial x} \int_{0}^{t} \frac{D_{\Phi_{V} S}}{k T} \nabla_{s} \mu(x, y, z, \tau) \times \\
& \times \int_{0}^{L_{0}}\left[\alpha_{2 \Phi_{V}}+\Phi_{1 V}(x, y, W, \tau)\right] d W d \tau+\Omega \frac{\partial}{\partial y} \int_{0}^{t} \nabla_{S} \mu(x, y, z, \tau) \int_{0}^{L_{L}}\left[\alpha_{2 \Phi_{V}}+\Phi_{1 V}(x, y, W, \tau)\right] d W \times \\
& \times \frac{D_{\Phi_{V} S}}{k T} d \tau+\int_{0}^{t} k_{V, V}(x, y, z, T) V^{2}(x, y, z, \tau) d \tau+\int_{0}^{t} k_{V}(x, y, z, T) V(x, y, z, \tau) d \tau+f_{\Phi_{V}}(x, y, z) .
\end{aligned}
$$

We determine average values of the second-order approximations of the considered concentrations by using standard relations $[25,31]$

$$
\alpha_{2 \rho}=\frac{1}{\Theta L_{x} L_{y} L_{z}} \int_{0}^{\Theta} \int_{0}^{L_{x} L_{y} L_{z}} \int_{0}\left[\rho_{0}(x, y, z, t)-\rho_{1}(x, y, z, t)\right] d z d y d x d t
$$

Substitution of the relations $(1 e),(3 e),(5 e)$ into relation (10) gives us possibility to obtain final relations for the required relations $\alpha_{2 \rho}$

$$
\begin{gathered}
\alpha_{2 C}=0, \alpha_{2 \Phi I}=0, \alpha_{2 \Phi V}=0, \alpha_{2 V}=\sqrt{\frac{\left(b_{3}+E\right)^{2}}{4 b_{4}^{2}}-4\left(F+\frac{\Theta a_{3} F+\Theta^{2} L_{x} L_{y} L_{z} b_{1}}{b_{4}}\right)}-\frac{b_{3}+E}{4 b_{4}}, \\
\alpha_{2 I}=\frac{C_{V}-\alpha_{2 V}^{2} S_{V V 00}-\alpha_{2 V}\left(2 S_{V V 01}+S_{I V 10}+\Theta L_{x} L_{y} L_{z}\right)-S_{V V 02}-S_{I V 11}}{S_{I V 01}+\alpha_{2 V} S_{I V 00}},
\end{gathered}
$$

where $b_{4}=\frac{1}{\Theta L_{x} L_{y} L_{z}} S_{I V 00}^{2} S_{V V 00}-\frac{1}{\Theta L_{x} L_{y} L_{z}} S_{V V 00}^{2} S_{I I 00}, b_{3}=-\frac{S_{I I 00}}{\Theta L_{x} L_{y} L_{z}} S_{V V 00}\left(2 S_{V V 01}+S_{I V 10}+\right.$

$$
\begin{aligned}
& \left.+\Theta L_{x} L_{y} L_{z}\right)+\frac{1}{\Theta L_{x} L_{y} L_{z}}\left(S_{I V 01}+2 S_{I I 10}+S_{I V 01}+\Theta L_{x} L_{y} L_{z}\right) S_{I V 00} S_{V V 00}+\frac{1}{\Theta L_{x} L_{y} L_{z}} S_{I V 00}^{2}\left(\Theta L_{x} L_{y} \times\right. \\
& \left.\times L_{z}+2 S_{V V 01}+S_{I V 10}\right)-S_{I V 00}^{2} S_{I V 10} / \Theta^{3} L_{x}^{3} L_{y}^{3} L_{z}^{3}, b_{2}=\frac{S_{I I 00} S_{V V 00}}{\Theta L_{x} L_{y} L_{z}}\left(S_{V V 02}+S_{I V 11}+C_{V}\right)-\left(\Theta L_{x} L_{y} L_{z}-\right. \\
& \left.-2 S_{V V 01}+S_{I V 10}\right)^{2}+\frac{S_{I V 01} S_{V V 00}}{\Theta L_{x} L_{y} L_{z}}\left(\Theta L_{x} L_{y} L_{z}+2 S_{I I 10}+S_{I V 01}\right)+\frac{S_{I V 00}}{\Theta L_{x} L_{y} L_{z}}\left(\Theta L_{x} L_{y} L_{z}+S_{I V 01}+2 S_{I I 10}+\right. \\
& \left.+2 S_{I V 01}\right)\left(2 S_{V V 01}+\Theta L_{x} L_{y} L_{z}+S_{I V 10}\right)-\left(C_{V}-S_{V V 02}-S_{I V 11}\right) \frac{S_{I V 00}^{2}}{\Theta L_{x} L_{y} L_{z}}-\frac{2}{\Theta L_{x} L_{y} L_{z}} S_{I V 10} S_{I V 00} S_{I V 01}+ \\
& +\frac{C_{I} S_{I V 00}^{2}}{\Theta^{2} L_{x}^{2} L_{y}^{2} L_{z}^{2}}, b_{1}=\frac{S_{I I 00}}{\Theta L_{x} L_{y} L_{z}}\left(S_{I V 11}+S_{V V 02}+C_{V}\right)\left(2 S_{V V 01}+S_{I V 10}+\Theta L_{x} L_{y} L_{z}\right)+\frac{S_{I V 01}}{\Theta L_{x} L_{y} L_{z}}\left(\Theta L_{x} \times\right. \\
& \left.\times L_{y} L_{z}+2 S_{I I 10}+S_{I V 01}\right)\left(2 S_{V V 01}+S_{I V 10}+\Theta L_{x} L_{y} L_{z}\right)-\frac{S_{I V 00}}{\Theta L_{x} L_{y} L_{z}}\left(3 S_{I V 01}+2 S_{I I 10}+\Theta L_{x} L_{y} L_{z}\right)\left(C_{V}-\right. \\
& \left.-S_{V V 02}-S_{I V 11}\right)+2 C_{I} S_{I V 00} S_{I V 01}-\frac{S_{I V 10} S_{I V 01}^{2}}{\Theta L_{x} L_{y} L_{z}}, b_{0}=\frac{S_{I I 00}}{\Theta L_{x} L_{y} L_{z}}\left(S_{I V 00}+S_{V V 02}\right)^{2}-\left(\Theta L_{x} L_{y} L_{z}+2 S_{I I 10}+\right. \\
& \left.+S_{I V 01}\right) \frac{S_{I V 01}}{\Theta L_{x} L_{y} L_{z}}\left(C_{V}-S_{I V 11}-S_{V V 02}\right)-\frac{S_{I V 01}}{\Theta L_{x} L_{y} L_{z}}\left(\Theta L_{x} L_{y} L_{z}+2 S_{I I 10}+S_{I V 01}\right)\left(C_{V}-S_{V V 02}-S_{I V 11}\right)+ \\
& +2 C_{I} S_{I V 01}^{2}, C_{I}=\alpha_{1 V} \frac{\alpha_{1 I}}{\Theta L_{x}} \frac{S_{I V 00}}{L_{y} L_{z}}+\frac{\alpha_{1 I}^{2} S_{I I 00}}{\Theta L_{x} L_{y} L_{z}}-\frac{S_{I I 20} S_{I I 20}}{\Theta L_{x} L_{y} L_{z}}-\frac{S_{I V 11}}{\Theta L_{x} L_{y} L_{z}}, C_{V}=\alpha_{1 I} \alpha_{1 V} S_{I V 00}-S_{V V 02}+ \\
& +\alpha_{1 V}^{2} S_{V V 00}-S_{I V 11}, E=\sqrt{8 y+\Theta^{2} \frac{a_{3}^{2}}{a_{4}^{2}}-4 \Theta \frac{a_{2}}{a_{4}}}, F=\frac{\Theta a_{2}}{6 a_{4}}+\sqrt[3]{\sqrt{r^{2}+s^{3}}-r}-\sqrt[3]{\sqrt{r^{2}+s^{3}}+r}, r=\frac{\Theta^{3} b_{2}}{24 b_{4}^{2}} \times
\end{aligned}
$$


Computer Applications: An International Journal (CAIJ), Vol.2, No.1, February 2015

$$
\begin{aligned}
& \times\left(4 b_{0}-\Theta L_{x} L_{y} L_{z} \frac{b_{1} b_{3}}{b_{4}}\right)-b_{0} \frac{\Theta^{2}}{8 b_{4}^{2}}\left(4 \Theta b_{2}-\Theta^{2} \frac{b_{3}^{2}}{b_{4}}\right)-\frac{\Theta^{3} b_{2}^{3}}{54 b_{4}^{3}}-L_{x}^{2} L_{y}^{2} L_{z}^{2} \frac{\Theta^{4} b_{1}^{2}}{8 b_{4}^{2}}, s=\frac{\Theta^{2}}{12 b_{4}^{2}}\left(4 b_{0} b_{4}-\right. \\
& \left.-\Theta L_{x} L_{y} L_{z} b_{1} b_{3}\right)-\Theta b_{2} / 18 b_{4} .
\end{aligned}
$$

Farther we determine solutions of the system of Eqs.(8). The solutions physically correspond to components of displacement vector. To determine components of displacement vector we used method of averaging of function corrections in standard form. Framework the approach we replace the above components in right sides of Eqs.(8) on their not yet known average values $\alpha_{i}$. The substitution leads to the following results

$$
\begin{gathered}
\rho(z) \frac{\partial^{2} u_{1 x}(x, y, z, t)}{\partial t^{2}}=-K(z) \beta(z) \frac{\partial T(x, y, z, t)}{\partial x}, \rho(z) \frac{\partial^{2} u_{1 y}(x, y, z, t)}{\partial t^{2}}=-K(z) \beta(z) \frac{\partial T(x, y, z, t)}{\partial y}, \\
\rho(z) \frac{\partial^{2} u_{1 z}(x, y, z, t)}{\partial t^{2}}=-K(z) \beta(z) \frac{\partial T(x, y, z, t)}{\partial z} .
\end{gathered}
$$

Integration of the left and right sides of the above equations on time $t$ leads to final relations for the first-order approximations of components of displacement vector in the following form

$$
\begin{aligned}
& u_{1 x}(x, y, z, t)=K(z) \frac{\beta(z)}{\rho(z)} \frac{\partial}{\partial x} \int_{0}^{t} \int_{0}^{\vartheta} T(x, y, z, \tau) d \tau d \vartheta-K(z) \frac{\beta(z)}{\rho(z)} \frac{\partial}{\partial x} \int_{0}^{\infty \vartheta} T(x, y, z, \tau) d \tau d \vartheta+u_{0 x}, \\
& u_{1 y}(x, y, z, t)=K(z) \frac{\beta(z)}{\rho(z)} \frac{\partial}{\partial} \int_{0}^{t} \int_{0}^{\vartheta} T(x, y, z, \tau) d \tau d \vartheta-K(z) \frac{\beta(z)}{\rho(z)} \frac{\partial}{\partial y} \int_{0}^{\infty \vartheta} T(x, y, z, \tau) d \tau d \vartheta+u_{0 y}, \\
& u_{1 z}(x, y, z, t)=K(z) \frac{\beta(z)}{\rho(z)} \frac{\partial}{\partial z} \int_{0}^{t} \int_{0}^{\vartheta} T(x, y, z, \tau) d \tau d \vartheta-K(z) \frac{\beta(z)}{\rho(z)} \frac{\partial}{\partial z} \int_{0}^{\infty \vartheta} T(x, y, z, \tau) d \tau d \vartheta+u_{0 z} .
\end{aligned}
$$

The second-order approximations and approximations with higher orders of components of displacement vector could be calculated by replacement of the required components in the Eqs. (8) on the following sums $\alpha_{i}+u_{i}(x, y, z, t)[25,31]$. This replacement leads to the following result

$$
\begin{gathered}
\rho(z) \frac{\partial^{2} u_{2 x}(x, y, z, t)}{\partial t^{2}}=\left\{K(z)+\frac{5 E(z)}{6[1+\sigma(z)]}\right\} \frac{\partial^{2} u_{1 x}(x, y, z, t)}{\partial x^{2}}+\left\{K(z)-\frac{E(z)}{3[1+\sigma(z)]}\right\} \times \\
\times \frac{\partial^{2} u_{1 y}(x, y, z, t)}{\partial x \partial y}+\frac{E(z)}{2[1+\sigma(z)]}\left[\frac{\partial^{2} u_{1 y}(x, y, z, t)}{\partial y^{2}}+\frac{\partial^{2} u_{1 z}(x, y, z, t)}{\partial z^{2}}\right]+\left\{K(z)+\frac{E(z)}{3[1+\sigma(z)]}\right\} \times \\
\times \frac{\partial^{2} u_{1 z}(x, y, z, t)}{\partial x \partial z}-K(z) \beta(z) \frac{\partial T(x, y, z, t)}{\partial x} \\
\begin{aligned}
& \rho(z) \frac{\partial^{2} u_{2 y}(x, y, z, t)}{\partial t^{2}}= \frac{E(z)}{2[1+\sigma(z)]}\left[\frac{\partial^{2} u_{1 y}(x, y, z, t)}{\partial x^{2}}+\frac{\partial^{2} u_{1 x}(x, y, z, t)}{\partial x \partial y}\right]-K(z) \beta(z) \frac{\partial T(x, y, z, t)}{\partial y}+ \\
&+\frac{\partial^{2} u_{1 y}(x, y, z, t)}{\partial y^{2}}\left\{\frac{5 E(z)}{12[1+\sigma(z)]}+K(z)\right\}+\frac{\partial}{\partial z}\left\{\frac{E(z)}{2[1+\sigma(z)]}\left[\frac{\partial u_{1 y}(x, y, z, t)}{\partial z}+\frac{\partial u_{1 z}(x, y, z, t)}{\partial y}\right]\right\}+ \\
&+\left\{K(z)-\frac{E(z)}{6[1+\sigma(z)]}\right\} \frac{\partial^{2} u_{1 y}(x, y, z, t)}{\partial y \partial z}+K(z) \frac{\partial^{2} u_{1 y}(x, y, z, t)}{\partial x \partial y}
\end{aligned}
\end{gathered}
$$


Computer Applications: An International Journal (CAIJ), Vol.2, No.1, February 2015

$$
\begin{gathered}
\rho(z) \frac{\partial^{2} u_{2 z}(x, y, z, t)}{\partial t^{2}}=\frac{E(z)}{2[1+\sigma(z)]}\left[\frac{\partial^{2} u_{1 z}(x, y, z, t)}{\partial x^{2}}+\frac{\partial^{2} u_{1 z}(x, y, z, t)}{\partial y^{2}}+\frac{\partial^{2} u_{1 x}(x, y, z, t)}{\partial x \partial z}+\frac{\partial^{2} u_{1 y}(x, y, z, t)}{\partial y \partial}\right]- \\
-K(z) \beta(z) \frac{\partial T(x, y, z, t)}{\partial z}+\frac{\partial}{\partial z}\left\{K(z)\left[\frac{\partial u_{1 x}(x, y, z, t)}{\partial x}+\frac{\partial u_{1 y}(x, y, z, t)}{\partial y}+\frac{\partial u_{1 x}(x, y, z, t)}{\partial z}\right]\right\}+ \\
+\frac{E(z)}{6[1+\sigma(z)]} \frac{\partial}{\partial z}\left\{\left[6 \frac{\partial u_{1 z}(x, y, z, t)}{\partial z}-\frac{\partial u_{1 x}(x, y, z, t)}{\partial x}-\frac{\partial u_{1 y}(x, y, z, t)}{\partial y}-\frac{\partial u_{1 z}(x, y, z, t)}{\partial z}\right]\right\} .
\end{gathered}
$$

Integration of left and right sides of the above equations on time $t$ leads to the following results

$$
\begin{aligned}
& u_{2 x}(x, y, z, t)=\frac{1}{\rho(z)}\left\{K(z)+\frac{5 E(z)}{6[1+\sigma(z)]}\right\} \frac{\partial^{2}}{\partial x^{2}} \iint_{0}^{t} \int_{0} u_{1 x}(x, y, z, \tau) d \tau d \vartheta+\frac{1}{\rho(z)}\left\{K(z)-\frac{E(z)}{3[1+\sigma(z)]}\right\} \times \\
& \times \frac{\partial^{2}}{\partial x \partial y} \int_{0}^{t} \int_{0}^{\vartheta} u_{1 y}(x, y, z, \tau) d \tau d \vartheta+\left[\frac{\partial^{2}}{\partial y^{2}} \int_{0}^{t} \int_{0}^{\vartheta} u_{1 y}(x, y, z, \tau) d \tau d \vartheta+\frac{\partial^{2}}{\partial z^{2}} \int_{0}^{t} \int_{0}^{\vartheta} u_{1 z}(x, y, z, \tau) d \tau d \vartheta\right] \times \\
& \times \frac{E(z)}{2 \rho(z)[1+\sigma(z)]}+\frac{1}{\rho(z)} \frac{\partial^{2}}{\partial x \partial z} \int_{00}^{t \vartheta} \int_{1 z}(x, y, z, \tau) d \tau d \vartheta\left\{K(z)+\frac{E(z)}{3[1+\sigma(z)]}\right\}-K(z) \frac{\beta(z)}{\rho(z)} \times \\
& \times \frac{\partial}{\partial x} \iint_{00}^{t} T(x, y, z, \tau) d \tau d \vartheta-\frac{1}{\rho(z)} \frac{\partial^{2}}{\partial x^{2}} \int_{00}^{\infty \vartheta} \int_{1 x}(x, y, z, \tau) d \tau d \vartheta\left\{K(z)+\frac{5 E(z)}{6[1+\sigma(z)]}\right\}-\frac{1}{\rho(z)}\{K(z)- \\
& \left.-\frac{E(z)}{3[1+\sigma(z)]}\right\} \frac{\partial^{2}}{\partial x \partial y} \int_{00}^{\infty \vartheta} \int_{1 y}(x, y, z, \tau) d \tau d \vartheta-\left[\frac{\partial^{2}}{\partial y^{2}} \int_{00}^{\infty \vartheta} \int_{1 y} u_{1 y}(x, y, z, \tau) d \tau d \vartheta+\frac{\partial^{2}}{\partial z^{2}} \int_{00}^{\infty \vartheta} \int_{1} u_{1 z}(x, y, z, \tau) d \tau d \vartheta\right] \times \\
& \times \frac{E(z)}{2 \rho(z)[1+\sigma(z)]}+K(z) \frac{\beta(z)}{\rho(z)} \frac{\partial}{\partial x} \int_{0}^{\infty \vartheta} \int_{0}^{\vartheta} T(x, y, z, \tau) d \tau d \vartheta-\frac{1}{\rho(z)} \frac{\partial^{2}}{\partial x \partial z} \int_{0}^{\infty \vartheta} \int_{1 z}(x, y, z, \tau) d \tau d \vartheta\{K(z)+ \\
& +E(z) / 3[1+\sigma(z)]\}+u_{0 x} \\
& u_{2 y}(x, y, z, t)=\frac{E(z)}{2 \rho(z)[1+\sigma(z)]}\left[\frac{\partial^{2}}{\partial x^{2}} \int_{0}^{t} \int_{0}^{\vartheta} u_{1 x}(x, y, z, \tau) d \tau d \vartheta+\frac{\partial^{2}}{\partial x \partial y} \int_{0}^{t} \int_{0}^{\vartheta} u_{1 x}(x, y, z, \tau) d \tau d \vartheta\right]+ \\
& +\frac{K(z)}{\rho(z)} \frac{\partial^{2}}{\partial x \partial y} \int_{0}^{t} \int_{0}^{\vartheta} u_{1 y}(x, y, z, \tau) d \tau d \vartheta+\frac{1}{\rho(z)}\left\{\frac{5 E(z)}{12[1+\sigma(z)]}+K(z)\right\} \frac{\partial^{2}}{\partial y^{2}} \int_{0}^{t \vartheta} \int_{0} u_{1 x}(x, y, z, \tau) d \tau d \vartheta+ \\
& +\frac{1}{2 \rho(z)} \frac{\partial}{\partial z}\left\{\frac{E(z)}{1+\sigma(z)}\left[\frac{\partial}{\partial z} \int_{0}^{t} \int_{0}^{\vartheta} u_{1 y}(x, y, z, \tau) d \tau d \vartheta+\frac{\partial}{\partial y} \int_{0}^{t} \int_{0}^{t} u_{1 z}(x, y, z, \tau) d \tau d \vartheta\right]\right\}-K(z) \frac{\beta(z)}{\rho(z)} \times \\
& \times \int_{0}^{t} \int_{0}^{t} T(x, y, z, \tau) d \tau d \vartheta+\frac{1}{\rho(z)}\left\{K(z)-\frac{E(z)}{6[1+\sigma(z)]}\right\} \frac{\partial^{2}}{\partial y \partial z} \int_{0}^{t} \int_{0}^{\vartheta} u_{1 y}(x, y, z, \tau) d \tau d \vartheta-\frac{E(z)}{2 \rho(z)} \times \\
& \times \frac{1}{1+\sigma(z)}\left\{\frac{\partial^{2}}{\partial x^{2}} \int_{0}^{\infty} \int_{0}^{\vartheta} u_{1 x}(x, y, z, \tau) d \tau d \vartheta+\frac{\partial^{2}}{\partial x \partial y} \int_{0}^{\infty \vartheta} \int_{0}^{\vartheta} u_{1 x}(x, y, z, \tau) d \tau d \vartheta\right\}-\int_{0}^{\infty \vartheta} T(x, y, z, \tau) d \tau d \vartheta \times \\
& \times K(z) \frac{\beta(z)}{\rho(z)}-\frac{K(z)}{\rho(z)} \frac{\partial^{2}}{\partial x \partial y} \int_{0}^{\infty \vartheta} \int_{0} u_{1 y}(x, y, z, \tau) d \tau d \vartheta-\frac{1}{\rho(z)} \frac{\partial^{2}}{\partial y^{2}} \int_{0}^{\infty} \int_{0}^{\vartheta} u_{1 x}(x, y, z, \tau) d \tau d \vartheta\{K(z)+ \\
& \left.+\frac{5 E(z)}{12[1+\sigma(z)]}\right\}-\frac{1}{2 \rho(z)} \frac{\partial}{\partial z}\left\{\frac{E(z)}{1+\sigma(z)}\left[\frac{\partial}{\partial z} \int_{0}^{\infty \vartheta} \int_{0}^{u_{1 y}}(x, y, z, \tau) d \tau d \vartheta+\frac{\partial}{\partial y} \int_{0}^{\infty \vartheta} \int_{0}^{\vartheta} u_{1 z}(x, y, z, \tau) d \tau d \vartheta\right]\right\}- \\
& -\frac{1}{\rho(z)}\left\{K(z)-\frac{E(z)}{6[1+\sigma(z)]}\right\} \frac{\partial^{2}}{\partial y \partial z} \int_{0}^{\infty \vartheta} \int_{0}^{\vartheta} u_{1 y}(x, y, z, \tau) d \tau d \vartheta+u_{0 y} \\
& \frac{\partial^{2} u_{z}(x, y, z, t)}{\partial t^{2}}=\left[\frac{\partial^{2}}{\partial x^{2}} \int_{0}^{\infty \vartheta} \int_{0}^{\vartheta} u_{1 z}(x, y, z, \tau) d \tau d \vartheta+\frac{\partial^{2}}{\partial y^{2}} \int_{0}^{\infty \vartheta} \int_{0}^{\vartheta} u_{1 z}(x, y, z, \tau) d \tau d \vartheta+\frac{\partial^{2}}{\partial x \partial z} \int_{0}^{\infty \vartheta} \int_{1 x}(x, y, z, \tau) d \tau d \vartheta+\right.
\end{aligned}
$$


Computer Applications: An International Journal (CAIJ), Vol.2, No.1, February 2015

$$
\begin{aligned}
& \left.+\frac{\partial^{2}}{\partial y \partial z} \int_{0}^{\infty \vartheta} \int_{0} u_{1 y}(x, y, z, \tau) d \tau d \vartheta\right] \frac{E(z)}{2 \rho(z)[1+\sigma(z)]}+\frac{1}{\rho(z)} \frac{\partial}{\partial z}\left\{\left[\frac{\partial}{\partial x} \int_{0}^{\infty \vartheta} \int_{0} u_{1 x}(x, y, z, \tau) d \tau d \vartheta+\right.\right. \\
& \left.\left.+\frac{\partial}{\partial y} \int_{0}^{\infty \vartheta} \int_{0} u_{1 x}(x, y, z, \tau) d \tau d \vartheta+\frac{\partial}{\partial z} \int_{0}^{\infty} \int_{0}^{\vartheta} u_{1 x}(x, y, z, \tau) d \tau d \vartheta\right] K(z)\right\}+\frac{1}{6 \rho(z)} \frac{\partial}{\partial z}\left\{\frac{E(z)}{1+\sigma(z)} \times\right. \\
& \times\left[6 \frac{\partial}{\partial z} \int_{0}^{\infty \vartheta} \int_{0}^{\infty} u_{1 z}(x, y, z, \tau) d \tau d \vartheta-\frac{\partial}{\partial x} \int_{0}^{\infty \vartheta} \int_{0}^{\vartheta} u_{1 x}(x, y, z, \tau) d \tau d \vartheta-\frac{\partial}{\partial y} \int_{0}^{\infty \vartheta} \int_{0}^{\vartheta} u_{1 y}(x, y, z, \tau) d \tau d \vartheta-\right. \\
& \left.\left.-\frac{\partial}{\partial z} \int_{0}^{\infty \vartheta} \int_{0} u_{1 z}(x, y, z, \tau) d \tau d \vartheta\right]\right\}-K(z) \frac{\beta(z)}{\rho(z)} \frac{\partial}{\partial z} \int_{0}^{\infty \vartheta} \int_{0}^{\infty} T(x, y, z, \tau) d \tau d \vartheta+u_{0 z} .
\end{aligned}
$$

Framework this paper we determine concentration of dopant, concentrations of radiation defects and components of displacement vector by using the second-order approximation framework method of averaging of function corrections. This approximation is usually enough good approximation to make qualitative analysis and to obtain some quantitative results. All obtained results have been checked by comparison with results of numerical simulations.

\section{Discussion}

In this section we analyzed influence of mismatch-induced stress on redistributions of dopant and radiation defects during annealing. The Figs. 2 and 3 show typical distributions of concentrations of dopant in heterostructures for diffusion and ion types of doping. All distributions in Figs. 2 and 3 have been calculated for larger value of dopant diffusion coefficient in doped area is larger in comparison with dopant diffusion coefficient in nearest areas. The figures show, that using inhomogeneity of heterostructure gives us possibility to increase sharpness of $p$ - $n$-junctions with increasing homogeneity of dopant distribution in doped part of epitaxial layer. Increasing of sharpness of $p-n$-junction leads to decreasing of switching time. Increasing of homogeneity of dopant distribution gives us possibility to decrease local overheats of materials during functioning of $p-n$ junction or decreasing of dimensions of the $p$ - $n$-junction for fixed maximal value of local overheat. It should be noted, that using the considered approach for manufacture a bipolar transistor leads to necessity to optimize annealing of dopant and/or radiation defects. Reason of this optimization is following. If annealing time is small, the dopant did not achieves any interfaces between materials of heterostructure. In this situation one can not find any modifications of distribution of concentration of dopant. Increasing of annealing time leads to increasing of homogeneity of dopant distribution. We optimize annealing time framework recently introduces approach [22,23,25,32-36]. Framework this criterion we approximate real distribution of concentration of dopant by step-wise function (see Figs. 4 and 5). Farther we determine optimal values of annealing time by minimization of the following mean-squared error

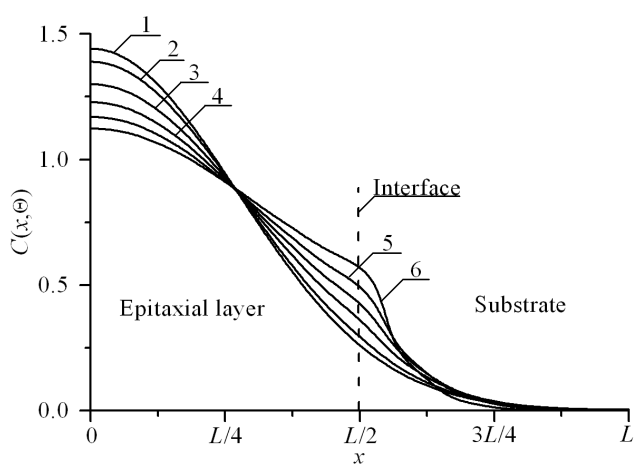


Fig.2. Typical distributions of concentration of dopant, which has been infused in heterostructure from Figs. 1 in direction, which is perpendicular to interface between epitaxial layer substrate. Increasing of number of curve corresponds to increasing of difference between values of dopant diffusion coefficient in layers of heterostructure under condition, when value of dopant diffusion coefficient in epitaxial layer is larger, than value of dopant diffusion coefficient in substrate

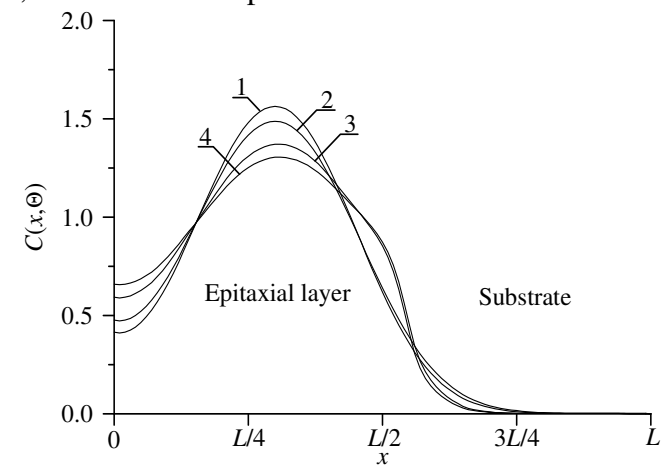

Fig.3. Typical distributions of concentration of dopant, which has been implanted in heterostructure from Figs. 1 in direction, which is perpendicular to interface between epitaxial layer substrate. Curves 1 and 3 corresponds to annealing time $\Theta=0.0048\left(L_{x}{ }^{2}+L_{y}{ }^{2}+L_{z}{ }^{2}\right) / D_{0}$. Curves 2 and 4 corresponds to annealing time $\Theta=0.0057\left(L_{x}{ }^{2}+L_{y}{ }^{2}+L_{z}{ }^{2}\right) / D_{0}$. Curves 1 and 2 corresponds to homogenous sample. Curves 3 and 4 corresponds to heterostructure under condition, when value of dopant diffusion coefficient in epitaxial layer is

larger, than value of dopant diffusion coefficient in substrate

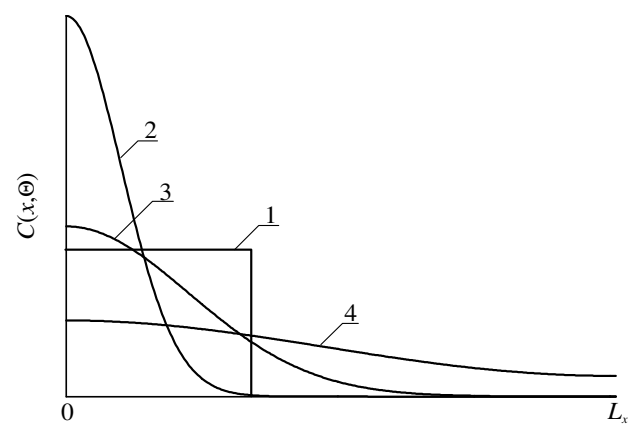

Fig. 4. Spatial distributions of dopant in heterostructure after dopant infusion. Curve 1 is idealized distribution of dopant. Curves 2-4 are real distributions of dopant for different values of annealing time. Increasing of number of curve corresponds to increasing of annealing time

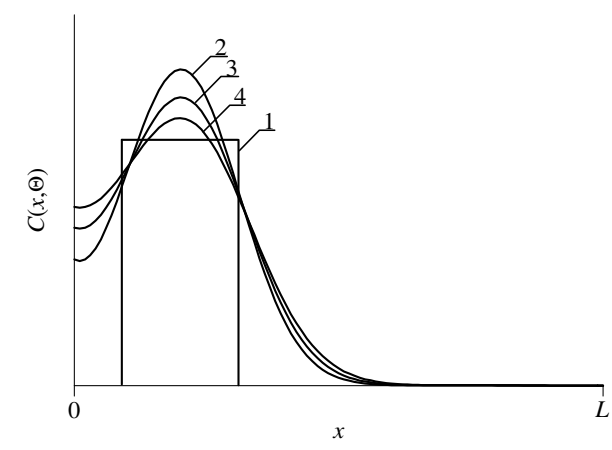

Fig. 5. Spatial distributions of dopant in heterostructure after ion implantation. Curve 1 is idealized distribution of dopant. Curves 2-4 are real distributions of dopant for different values of annealing time. Increasing of number of curve corresponds to increasing of annealing time 


$$
U=\frac{1}{L_{x} L_{y} L_{z}} \int_{0}^{L_{x}} \int_{0}^{L_{y}} \int_{0}^{L_{z}}[C(x, y, z, \Theta)-\psi(x, y, z)] d z d y d x
$$

where $\psi(x, y, z)$ is the approximation function. Dependences of optimal values of annealing time on parameters are presented on Figs. 6 and 7 for diffusion and ion types of doping, respectively. It should be noted, that it is necessary to anneal radiation defects after ion implantation. One could find spreading of concentration of distribution of dopant during this annealing. In the ideal case distribution of dopant achieves appropriate interfaces between materials of heterostructure during annealing of radiation defects. If dopant did not achieves any interfaces during annealing of radiation defects, it is practicably to additionally anneal the dopant. In this situation optimal value of additional annealing time of implanted dopant is smaller, than annealing time of infused dopant.

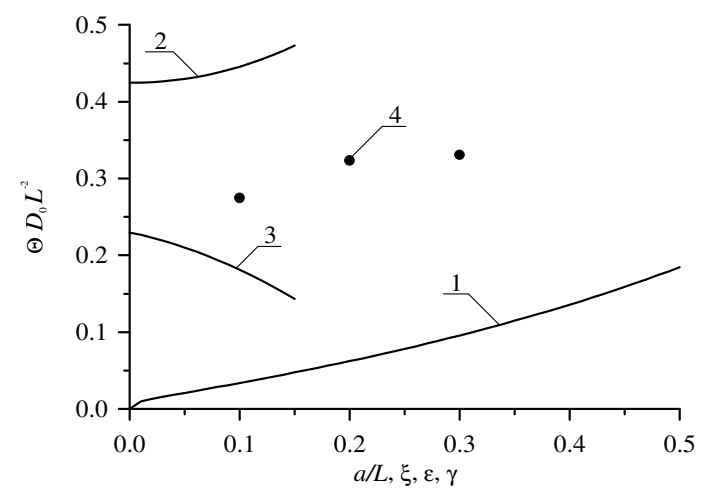

Fig.6. Dependences of dimensionless optimal annealing time for doping by diffusion on several parameters.

Curve 1 is the dependence of dimensionless optimal annealing time on the relation $a / L$ and $\xi=\gamma=0$ for equal to each other values of dopant diffusion coefficient in all parts of heterostructure. Curve 2 is the dependence of dimensionless optimal annealing time on value of parameter $\varepsilon$ for $a / L=1 / 2$ and $\xi=\gamma=0$. Curve 3 is the dependence of dimensionless optimal annealing time on value of parameter $\xi$ for $a / L=1 / 2$ and $\varepsilon=\gamma$ $=0$. Curve 4 is the dependence of dimensionless optimal annealing time on value of parameter $\gamma$ for $a / L=1 / 2$ and $\varepsilon=\xi=0$

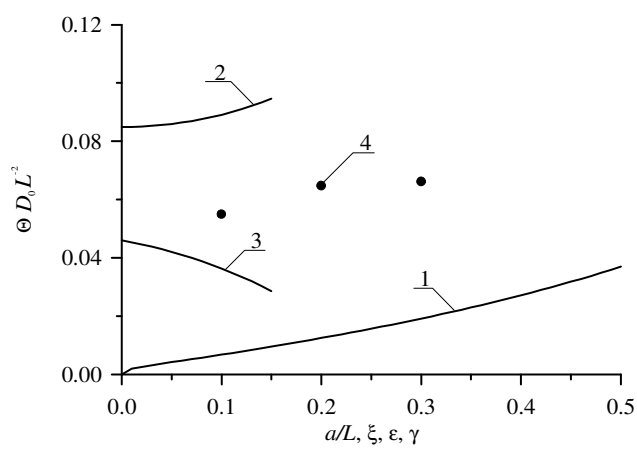

Fig.7. Dependences of dimensionless optimal annealing time for doping by ion implantation on several parameters. Curve 1 is the dependence of dimensionless optimal annealing time on the relation $a / L$ and $\xi=$ $\gamma=0$ for equal to each other values of dopant diffusion coefficient in all parts of heterostructure. Curve 2 is the dependence of dimensionless optimal annealing time on value of parameter $\varepsilon$ for $a / L=1 / 2$ and $\xi=\gamma=0$. Curve 3 is the dependence of dimensionless optimal annealing time on value of parameter $\xi$ for $a / L=1 / 2$ and $\varepsilon=\gamma=0$. Curve 4 is the dependence of dimensionless optimal annealing time on value of parameter $\gamma$ for $a / L=1 / 2$ and $\varepsilon=\xi=0$

Farther we analyzed influence of relaxation of mechanical stress on distribution of dopant in doped areas of heterostructure. Under following condition $\varepsilon_{0}<0$ one can find compression of distribution of concentration 
Computer Applications: An International Journal (CAIJ), Vol.2, No.1, February 2015

of dopant near interface between materials of heterostructure. Contrary (at $\varepsilon_{0}>0$ ) one can find spreading of distribution of concentration of dopant in this area. This changing of distribution of concentration of dopant could be at least partially compensated by using laser annealing [33]. This type of annealing gives us possibility to accelerate diffusion of dopant and another processes in annealed area due to inhomogenous distribution of temperature and Arrhenius law. Accounting relaxation of mismatch-induced stress in heterostructure could leads to changing of optimal values of annealing time.

\section{Conclusions}

In this paper we analyzed influence of relaxation of mismatch-induced stress in heterostructure on distributions of concentrations of dopants in manufactured in this heterostructure transistors. At the same time in this paper we introduce an approach to increase compactness of multiemitter transistor. The approach based on doping by diffusion or ion implantation of required parts of dopants and/or radiation defects.

\section{Acknowledgments}

This work is supported by the contract 11.G34.31.0066 of the Russian Federation Government, grant of Scientific School of Russia, the agreement of August 27, 2013 № 02.B.49.21.0003 between The Ministry of education and science of the Russian Federation and Lobachevsky State University of Nizhni Novgorod and educational fellowship for scientific research of Nizhny Novgorod State University of Architecture and Civil Engineering.

\section{REFERENCES}

[1] I.P. Stepanenko. Basis of Microelectronics (Soviet Radio, Moscow, 1980).

[2] A.G. Alexenko, I.I. Shagurin. Microcircuitry. Moscow: Radio and communication, 1990.

[3] Z.Yu. Gotra, Technology of microelectronic devices (Radio and communication, Moscow, 1991).

[4] N.A. Avaev, Yu.E. Naumov, V.T. Frolkin. Basis of microelectronics (Radio and communication, Moscow, 1991).

[5] V.I. Lachin, N.S. Savelov. Electronics. Rostov-on-Don: Phoenix, 2001.

[6] A. Polishscuk. analog integrated circuits Anadigm: the whole range of analog electronics on a single chip. The first acquaintance. Modern Electronics. Issue 12. P. 8-11 (2004).

[7] G. Volovich. Modern chips UM3Ch class D manufactured by firm MPS. Modern Electronics. Issue 2. P. 10-17 (2006).

[8] A. Kerentsev, V. Lanin, Constructive-technological features of MOSFET- transistors. Power Electronics. Issue 1. P. 34 (2008).

[9] A.O. Ageev, A.E. Belyaev, N.S. Boltovets, V.N. Ivanov, R.V. Konakova, Ya.Ya. Kudrik, P.M. Litvin, V.V. Milenin, A.V. Sachenko. Au-TiBx-n-6H-SiC Schottky barrier diodes: the features of current flow in rectifying and nonrectifying contacts. Semiconductors. Vol. 43 (7). P. 897-903 (2009).

[10] Jung-Hui Tsai, Shao-Yen Chiu, Wen-Shiung Lour, Der-Feng Guo. High-performance InGaP/GaAs pnp $\delta$-doped heterojunction bipolar transistor. Semiconductors. Vol. 43 (7). P. 971-974 (2009).

[11] E.I. Gol'dman, N.F. Kukharskaya, V.G. Naryshkina, G.V. Chuchueva. The manifestation of excessive centers of the electron-hole pair generation, appeared as a result to field and thermal stresses, and their subsequent annihilation in the dynamic current-voltage characteristics of Si-MOS-structures with the ultrathin oxide Semiconductors. Vol. 45 (7). P. 974-979 (2011).

[12] T.Y. Peng, S.Y. Chen, L.C. Hsieh C.K. Lo, Y.W. Huang, W.C. Chien, Y.D. Yao. Impedance behavior of spin-valve transistor. J. Appl. Phys. Vol. 99 (8). P. 08H710-08H712 (2006).

[13] W. Ou-Yang, M. Weis, D. Taguchi, X. Chen, T. Manaka, M. Iwamoto. Modeling of threshold voltage in pentacene organic field-effect transistors J. Appl. Phys. Vol. 107 (12). P. 124506-124510 (2010).

[14] J. Wang, L. Wang, L. Wang, Z. Hao, Yi Luo, A. Dempewolf, M. M ller, F. Bertram, J rgen Christen. An improved carrier rate model to evaluate internal quantum efficiency and analyze efficiency droop origin of InGaN based light-emitting diodes. J. Appl. Phys. Vol. 112 (2). P. 023107-023112 (2012).

[15] O.V. Alexandrov, A.O. Zakhar'in, N.A. Sobolev, E.I. Shek, M.M. Makoviychuk, E.O. Parshin. Formation of donor centers after annealing of dysprosium and holmium implanted silicon. Semiconductors. Vol. 32 (9). P. 1029-1032 (1998).

[16] I.B. Ermolovich, V.V. Milenin, R.A. Red'ko, S.M. Red'ko. Features of recombination processes in $\mathrm{CdTe}$ films, preparing at different temperature conditions and further annealing. Semiconductors. Vol. 43 (8). P. 1016-1020 (2009). 
Computer Applications: An International Journal (CAIJ), Vol.2, No.1, February 2015

[17] P. Sinsermsuksakul, K. Hartman, S.B. Kim, J. Heo, L. Sun, H.H. Park, R. Chakraborty, T. Buonassisi, R.G. Gordon. Enhancing the efficiency of SnS solar cells via band-offset engineering with a zinc oxysulfide buffer layer. Appl. Phys. Lett. Vol. 102 (5). P. 053901-053905 (2013).

[18] J.G. Reynolds, C.L. Reynolds, Jr.A. Mohanta, J.F. Muth, J.E. Rowe, H.O. Everitt, D.E. Aspnes. Shallow acceptor complexes in p-type ZnO Appl. Phys. Lett. Vol. 102 (15). P. 152114-152118 (2013).

[19] K.K. Ong, K.L. Pey, P.S. Lee, A.T.S. Wee, X.C. Wang, Y.F. Chong, Dopant distribution in the recrystallization transient at the maximum melt depth induced by laser annealing Appl. Phys. Lett. 89 (17), 172111-172114 (2006).

[20] H.T. Wang, L.S. Tan, E. F. Chor. Pulsed laser annealing of Be-implanted GaN. J. Appl. Phys. 98 (9), 094901-094905 (2006).

[21] Yu.V. Bykov, A.G. Yeremeev, N.A. Zharova, I.V. Plotnikov, K.I. Rybakov, M.N. Drozdov, Yu.N. Drozdov, V.D. Skupov. Diffusion processes in semiconductor structures during microwave annealing. Radiophysics and Quantum Electronics. Vol. 43 (3). P. 836-843 (2003).

[22] E.L. Pankratov. Dopant diffusion dynamics and optimal diffusion time as influenced by diffusioncoefficient nonuniformity. Russian Microelectronics. 2007. V.36 (1). P. 33-39.

[23] E.L. Pankratov. Redistribution of dopant during annealing of radiative defects in a multilayer structure by laser scans for production an implanted-junction rectifiers. Int. J. Nanoscience. Vol. 7 (4-5). P. 187-197 (2008).

[24] V.V. Kozlivsky. Modification of semiconductors by proton beams (Nauka, Sant-Peterburg, 2003, in Russian).

[25] E.L. Pankratov, E.A. Bulaeva. Doping of materials during manufacture p-n-junctions and bipolar transistors. Analytical approaches to model technological approaches and ways of optimization of distributions of dopants. Reviews in Theoretical Science. Vol. 1 (1). P. 58-82 (2013).

[26] Y.W. Zhang, A.F. Bower. Numerical simulations of island formation in a coherent strained epitaxial thin film system. Journal of the Mechanics and Physics of Solids. Vol. 47 (11). P. 2273-2297 (1999).

[27] L.D. Landau, E.M. Lefshits. Theoretical physics. 7 (Theory of elasticity) (Physmatlit, Moscow, 2001, in Russian).

[28] E.I. Zorin, P.V. Pavlov and D.I. Tetelbaum. Ion doping of semiconductors. Moscow: Energy, 1975.

[29] P.M. Fahey, P.B. Griffin, J.D. Plummer. Point defects and dopant diffusion in silicon. Rev. Mod. Phys. 1989. V. 61. № 2. P. 289-388.

[30] V.L. Vinetskiy, G.A. Kholodar', Radiative physics of semiconductors. ("Naukova Dumka", Kiev, 1979, in Russian).

[31] Yu.D. Sokolov. About the definition of dynamic forces in the mine lifting Applied Mechanics. Applied Mechanics. Vol.1 (1). P. 23-35 (1955).

[32] E.L. Pankratov, E.A. Bulaeva. Decreasing of quantity of radiation defects in an implanted-junction rectifiers by using overlayers. Int. J. Micro-Nano Scale Transp. Vol. 3 (3). P. 119-130 (2012).

[33] E.L. Pankratov. Increasing of the sharpness of p-n-junctions by laser pulses Nano. Vol. 6 (1). P. 31-40 (2011).

[34] E.L. Pankratov, E.A. Bulaeva. Application of native inhomogeneities to increase compactness of vertical field -effect transistors. J. Comp. Theor. Nanoscience. Vol. 10 (4). P. 888-893 (2013).

[35] E.L. Pankratov, E.A. Bulaeva. Optimization of doping of heterostructure during manufacturing of p-in-diodes. Nanoscience and Nanoengineering. Vol. 1 (1). P. 7-14 (2013).

[36] E.L. Pankratov, E.A. Bulaeva. An approach to manufacture of bipolar transistors in thin film structures. On the method of optimization. Int. J. Micro-Nano Scale Transp. Vol. 4 (1). P. 17-31 (2014).

\section{Authors:}

Pankratov Evgeny Leonidovich was born at 1977. From 1985 to 1995 he was educated in a secondary school in Nizhny Novgorod. From 1995 to 2004 he was educated in Nizhny Novgorod State University: from 1995 to 1999 it was bachelor course in Radiophysics, from 1999 to 2001 it was master course in Radiophysics with specialization in Statistical Radiophysics, from 2001 to 2004 it was PhD course in Radiophysics. From 2004 to 2008 E.L. Pankratov was a leading technologist in Institute for Physics of Microstructures. From 2008 to 2012 E.L. Pankratov was a senior lecture/Associate Professor of Nizhny Novgorod State University of Architecture and Civil Engineering. Now E.L. Pankratov is in his Full Doctor course in Radiophysical Department of Nizhny Novgorod State University. He has 105 published papers in area of his researches.

Bulaeva Elena Alexeevna was born at 1991. From 1997 to 2007 she was educated in secondary school of village Kochunovo of Nizhny Novgorod region. From 2007 to 2009 she was educated in boarding school 
Computer Applications: An International Journal (CAIJ), Vol.2, No.1, February 2015

"Center for gifted children". From 2009 she is a student of Nizhny Novgorod State University of Architecture and Civil Engineering (spatiality "Assessment and management of real estate"). At the same time she is a student of courses "Translator in the field of professional communication" and "Design (interior art)" in the University. E.A. Bulaeva was a contributor of grant of President of Russia (grant № MK548.2010.2). She has 52 published papers in area of her researches. 